\title{
Relation tutorale et configurations de participation à l'interaction : Analyse de l'accompagnement des stagiaires dans le champ de la petite enfance
}

Mentoring trainees through interactional participatory configurations: An analysis of guidance practices in an early childhood education training program

\section{Laurent Filliettaz, Vanessa Rémery et Dominique Trébert}

\section{OpenEdition}

\section{Journals}

Édition électronique

URL : http://journals.openedition.org/activites/288

DOI : 10.4000/activites.288

ISSN : 1765-2723

Éditeur

ARPACT - Association Recherches et Pratiques sur les ACTivités

\section{Référence électronique}

Laurent Filliettaz, Vanessa Rémery et Dominique Trébert, « Relation tutorale et configurations de participation à l'interaction : Analyse de l'accompagnement des stagiaires dans le champ de la petite enfance », Activités [En ligne], 11-1 | Avril 2014, mis en ligne le 15 avril 2014, consulté le 19 avril 2019. URL : http://journals.openedition.org/activites/288; DOI : 10.4000/activites.288

\section{(c) (i) $\odot$}

Activités est mis à disposition selon les termes de la licence Creative Commons Attribution - Pas d'Utilisation Commerciale - Pas de Modification 4.0 International. 


\title{
Relation tutorale et configurations de participation à l'interaction :
}

\section{Analyse de l'accompagnement des stagiaires dans le champ de la petite enfance}

\author{
Laurent Filliettaz \\ Équipe Interaction \& Formation, \\ Université de Genève, FAPSE, 40 bd du pont d'Arve, 1211 Genève 4, Suisse \\ Laurent.Filliettaz@unige.ch

\section{Vanessa Rémery} \\ Équipe Interaction \& Formation, \\ Université de Genève, FAPSE, 40 bd du pont d'Arve, 1211 Genève 4, Suisse \\ Vanessa.Remery@unige.ch

\section{Dominique Trébert} \\ Équipe Interaction \& Formation, \\ Université de Genève, FAPSE, 40 bd du pont d'Arve, 1211 Genève 4, Suisse \\ Dominique.Trebert@unige.ch
}

En hommage à Patrick Kunégel, trop vite disparu.

\begin{abstract}
Mentoring trainees through interactional participatory configurations: An analysis of guidance practices in an early childhood education training program. . Recent literature in the field of workplace learning has stressed the importance of guidance in the process of learning in and through professional practice. Workers do not learn just by performing specific tasks on their own; they learn when they are afforded adequate resources and when more experienced workers are able to share their knowledge and skills and assist them in their work. In line with this body of research, the paper addresses the topic of guidance at work in a specific context: that of early childhood educators and their vocational training. By adopting an interactional and multimodal perspective on guidance, the paper investigates how training opportunities are provided by vocational trainers in the context of internships. What constitutes the "practice of mentoring" in early childhood institutions? Through what sorts of "action" is guidance provided and accomplished in naturally occurring educational practices? How do mentors provide students with participation opportunities in naturally occurring educational practices? How do students engage with these participation opportunities and make use of the resources afforded to them? In this paper, three distinct interactional participatory configurations are identified, defined and empirically illustrated: i) observation, ii) joint-action, and iii) display. These configurations stress both the complexity and the dynamic nature of training practices as they occur under working conditions
\end{abstract}

KEY WORDS

mentoring, guidance, workplace learning, interaction, participation, scaffolding 


\section{1.- L'activité tutorale sur le lieu de travail}

La confrontation à des situations de travail réelles et l'intégration dans un collectif de travail occupent une place importante dans les dispositifs de formation qui préparent les jeunes à l'acquisition d'un métier par la voie professionnelle. Du point de vue des concepteurs de ces dispositifs, l'expérience du travail en entreprise est à la fois perçue comme un vecteur de socialisation professionnelle et comme un moyen de développer les compétences requises par les référentiels de formation. Dans le contexte d'un développement marqué des dispositifs de formation par l'apprentissage et fondés sur l'alternance (Veillard \& Kouamé Kouassi, 2012), la qualité de l'accompagnement proposé aux apprenants en situation de travail revêt un enjeu social et éducatif de première importance. Les travaux de recherche conduits récemment dans le champ de la sociologie des parcours de formation des jeunes soulignent de manière unanime le rôle primordial de l'accompagnement tutoral pour la réussite des transitions vers le monde professionnel. Ces travaux indiquent que la disponibilité des tuteurs et la qualité de leurs interactions avec les apprenants constituent des facteurs non négligeables de la réussite des apprentissages. Ces travaux mettent en évidence la part importante des abandons et des arrêts prématurés des parcours de formation dans des situations problématiques et détériorées (Duc, 2012; Lamamra \& Masdonati, 2009 ; Stalder \& Nägele, 2011).

Paradoxalement, il existe à ce jour peu de travaux de recherche qui portent spécifiquement sur l'activité des tuteurs en entreprise. Dans les organisations, l'accompagnement tutoral tend à se confondre avec l'activité de travail elle-même et constitue une pratique largement transparente, que les travailleurs sont appelés à réaliser spontanément et sans réelle reconnaissance sociale. Comme le relèvent Barbier (1996) et Kunégel (2011), une conception par ailleurs normative et prescriptive domine souvent dans la littérature sur le tutorat. On tend à prescrire les attitudes et comportements des tuteurs, sans pour autant s'intéresser pleinement à la dynamique et à l'organisation de leur activité.

Le présent article vise précisément à contribuer à résoudre ce paradoxe dans un champ empirique particulier, celui de la formation professionnelle des éducatrices ${ }^{1}$ de l'enfance. En Suisse, dans le canton de Genève, la formation des éducatrices de l'enfance est conçue selon une logique d'alternance et inclut, durant les trois années de formation, divers stages, dont la durée oscille entre huit semaines et neuf mois. Réalisés au sein de diverses institutions de la petite enfance (IPE), ces stages sont accompagnés par des éducatrices expérimentées, qui endossent à l'égard des stagiaires une fonction de «référente professionnelle». Les référentes professionnelles assument, en plus de leur fonction éducative auprès des enfants, une mission d'encadrement et d'accompagnement auprès des stagiaires. Il est attendu des référentes professionnelles qu'elles assurent un accompagnement pédagogique à l'attention des stagiaires et qu'elles partagent avec ceux-ci «les gestes du métier». Dans ce contexte, l'article propose de mieux comprendre comment s'y prennent les éducatrices endossant une fonction de référente professionnelle pour accompagner des stagiaires sur la place de travail. Dans la continuité des travaux récents produits dans le champ des sciences du travail et de la formation sur le tutorat, l'article propose d'étudier la manière dont les référentes professionnelles investissent leur rôle de tutrices auprès des apprenants qu'elles forment, dans les situations de travail reposant sur la conduite d'une activité éducative auprès des enfants. Nous examinerons plus spécifiquement les processus interactionnels au moyen desquels des formes d'accompagnement sont établies, négociées et reconfigurées dans le cours de l'activité de travail. Ceci nous amènera à caractériser comment, dans le quotidien du travail en crèche, les référentes professionnelles aménagent pour les stagiaires des

1 Par souci d'allègement et pour respecter le fait que cette profession est majoritairement représentée par les femmes, le féminin sera ici préféré au masculin, en dépit des règles de grammaire. L'usage du féminin n'occulte cependant pas le fait qu'il existe également des éducateurs dans le champ de la petite enfance. 
opportunités de participation à des activités de travail. Et réciproquement, comment les stagiaires investissent ces opportunités de participation pour expérimenter, sous différentes facettes, les compétences professionnelles visées dans leur parcours de formation.

À partir de la mise en perspective de quelques travaux existants portant sur l'activité tutorale en situation de travail, nous explicitons d'abord l'orientation théorique et méthodologique que nous privilégions dans nos propres recherches et les apports possibles d'une perspective située, interactionnelle et multimodale au domaine d'étude de l'activité tutorale (2). La notion de «configuration de participation » est ensuite introduite, en référence aux apports de la micro-sociologie goffmannienne et des théories linguistiques de l'interaction (3). À partir d'un matériau audio-vidéo documentant des situations de formation par le travail en crèche, nous proposons de dégager différentes caractéristiques des interactions tutorales qui s'y déploient. Trois configurations de participation sont identifiées et illustrées par nos données, qui permettent de saisir la diversité des formats par lesquels les référentes endossent une fonction d'accompagnement tutoral dans le quotidien de leur travail (4). Ces configurations de participation sont finalement mises en perspectives et interrogées à l'aune des spécificités du travail éducatif en crèche et des enjeux de reconnaissance sociale qui caractérisent ce champ professionnel particulier (5).

\section{2.- Explorer les spécificités de l'accompagnement tutoral en situation de travail}

Dans le contexte paradoxal d'une activité à la fois perçue comme décisive mais encore relativement méconnue et peu documentée par la recherche, un ensemble de travaux récents portent malgré tout une attention croissante à la problématique de l'accompagnement tutoral en formation professionnelle. Conduits dans les domaines de la didactique professionnelle francophone ou du courant anglo-saxon du Workplace Learning, ces travaux proposent une vision convergente en ce qu'ils abordent l'accompagnement tutoral d'une part comme une forme organisée d'activité et d'autre part comme une réalité collective et résultant des influences conjointes des tuteurs et des apprenants.

S'intéresser à l'activité tutorale et chercher à en comprendre les spécificités revient, pour la didactique professionnelle, à prendre ses distances avec une conception scolaire de la formation fondée principalement sur la transmission de contenus de savoirs théoriques. Comme le relève Savoyant (1995, 1996), l'activité d'un tuteur en entreprise diffère de celle d'un enseignant au sens où les savoirs transmis ne correspondent pas à des contenus d'informations décontextualisés du travail mais résultent d'une imbrication profonde et complexe avec des activités de travail. Se pose alors la question des rapports entre les dimensions «constructives » et «productives » de l'activité de travail (Rabardel, 2005) du fait que les exigences de production s'imposent aux situations de tutorat (Mayen, 2002 ; Olry \& Cuvillier, 2007 ; Olry-Louis \& Olry, 2011). À ce propos, Kunégel $(2005,2011)$ relève que l'activité tutorale se déploie selon des formes, des degrés et des configurations qui varient. À partir d'un travail empirique minutieux réalisé dans le champ de la mécanique automobile, Kunégel a mis en évidence une typologie des formes d'interactions tutorales sur la place de travail : la sélection de la tâche, la consigne, le laisser-faire, le guidage, la monstration et l'évaluation. Ces formes d'interaction tutorale évoluent dans le temps, en fonction notamment du degré d'autonomie de l'apprenant dans la réalisation d'une tâche et des modes d'organisation de la dyade tuteur-apprenant. S'appuyant sur la notion de « format » proposée par Bruner (1983) pour étudier les interactions de tutelle, Kunégel a identifié des régularités dans l'organisation séquentielle des formats et propose à cet égard un "modèle diachronique » de l'activité tutorale, consistant à identifier six configurations que les acteurs accomplissent successivement dans les parcours de formation: familiarisation, familiarisation avancée, transmission, mise au travail assistée, mise au travail semi-assistée et mise au travail. Cette échelle de configurations met en évidence le processus 
d'autonomisation progressive de l'apprenant d'une part et la transformation du type de guidage déployé par le tuteur d'autre part.

Cette modélisation n'est pas sans rappeler des thèses largement diffusées dans le champ des approches anthropologiques de la formation, au sein desquelles l'apprentissage est envisagé comme une participation progressive aux activités d'une communauté de pratique (Lave \& Wenger, 1991 ; Wenger, 1998). Ces travaux ont montré l'importance des phénomènes de socialisation et d'acculturation professionnelle dans les apprentissages au travail. Ils ont permis également de relever que toutes les situations de travail ne permettent pas systématiquement d'engager une trajectoire relevant de la participation périphérique légitime, au cours de laquelle les novices se confrontent progressivement à des pratiques de complexité croissante et font l'objet d'une reconnaissance identitaire progressive. En effet, comme le relèvent Fuller et Unwin (2003), les environnements de travail ne sont pas tous « expansifs» du point de vue des apprentissages. Il existe des situations « restrictives » où les apprentis demeurent durablement à la marge des communautés de pratique dans lesquelles ils aspirent à être reconnus. Dans cette perspective, les modalités de l'accompagnement tutoral sont perçues comme des ingrédients déterminants des apprentissages au travail. Ces modalités régissent à la fois les conditions d'accès des apprenants aux pratiques professionnelles, les ressources mises à leur disposition pour les réaliser, et les logiques de reconnaissance et de positionnement identitaire qui les soustendent. Dans la perspective d'une meilleure connaissance des facteurs sur lesquels repose l'efficacité des apprentissages au travail, les travaux de Billett $(2001,2009)$ dans le champ du workplace learning montrent que la qualité des apprentissages est façonnée par les «modalités de participation en situation de travail » (workplace participatory practices), qui reposent sur deux types de facteurs. D'une part, la façon dont l'environnement professionnel « offre » la possibilité aux apprenants de participer à des activités et des interactions avec les autres membres de la communauté. Ces ressources sont désignées comme des «affordances ». D'autre part, la manière dont les apprenants font usage de ces ressources et s'engagent personnellement dans les activités et les formes d'accompagnement disponibles sur la place de travail. Ces facteurs individuels relèvent de ce que Billett désigne comme «l'engagement». «Affordance» et «engagement» correspondent ainsi aux deux ingrédients d'une dualité constitutive des apprentissages professionnels. À la lumière de ce modèle, la question de l'accompagnement tutoral occupe une place à la fois centrale et relative. En tant que ressource accessible dans les environnements de travail, la disponibilité des tuteurs et des experts constitue un ingrédient déterminant des «affordances » du contexte professionnel. Mais ces ressources ne valent que si les apprenants en font usage et s'ils s'engagent dans les positions réciproques configurées par les tuteurs.

En continuité avec ces travaux, nous privilégions, pour notre part, un point de vue méthodologique ancré dans le champ de l'analyse du travail, mais qui prend ses distances avec des méthodes d'investigation qui s'intéressent de manière prépondérante à la réalisation et à l'interprétation d'entretiens réalisés avec les travailleurs (Clot, 2000 ; Theureau, 2000 ; Vermersch, 1994). Sans pour autant minimiser l'importance des représentations subjectives élaborées par les acteurs à propos de leur activité, notre démarche relève avant tout d'une description ethnographique de l'activité, qui s'appuie sur l'immersion du chercheur dans les situations de travail réelles ainsi que sur un recueil d'enregistrements audio-vidéo de situations naturelles de travail et de formation. L'objectif d'une telle démarche est de documenter la dynamique de l'activité au plan collectif, dans son déploiement séquentiel, et dans les significations en actes que véhiculent les contributions des participants au moment de s'engager conjointement dans l'interaction (Heath, Knoblauch, \& Luff, 2000 ; Mondada, 2006). Dans cette perspective, les processus d'accompagnement et de formation qui se déploient en situation de travail sont appréhendés comme des activités situées, en perpétuelle transformation, et accomplies collectivement dans l'interaction, au moyen d'une combinaison de multiples ressources sémiotiques. Cette approche peut être ainsi qualifiée de 
située, interactionnelle et multimodale (Duc, 2012; Filliettaz, 2009, 2010, 2011a, 2011b ; Filliettaz, de Saint-Georges \& Duc, 2008, 2012). La perspective adoptée est située en ce qu'elle propose d'approcher les processus d'accompagnement tutoral comme des accomplissements pratiques, des activités, qui prennent place dans des environnements matériels et culturels singuliers. Elle est interactionnelle au sens où elle est centrée avant tout sur l'étude des interactions entre tuteurs et apprenants et au moyen desquelles ils s'engagent dans l'activité de travail. Ces interactions sont envisagées comme des processus de coordination par lesquels les participants rendent publiquement manifeste leur engagement dans des pratiques sociales singulières. Ce sont les conditions de cette coordination et de cet accomplissement qui constituent l'objet de l'analyse et qui sont à même d'éclairer l'organisation des pratiques sociales étudiées. Enfin, la perspective adoptée est dite multimodale au sens où les significations mises en circulation dans l'interaction ne découlent plus seulement des productions verbales mais reposent également sur une vaste palette d'autres ressources sémiotiques (la gestualité, les mimiques faciales, les mouvements corporels, les déplacements dans l'espace, les manipulations d'objets matériels et symboliques, etc.). Et surtout, ces significations résultent d'une combinaison et d'une agrégation permanente de ces multiples ressources dans des contextes d'usages singuliers. Adopter une perspective multimodale sur les interactions tutorales revient donc pour nous à observer non seulement comment les participants à l'interaction se coordonnent et ajustent leur engagement pour agir collectivement, mais encore à repérer les sortes de ressources sémiotiques mobilisées à cette fin et la manière dont celles-ci sont mises en œuvre dans les différentes pratiques de formation observables au sein des institutions du travail (Filliettaz, à paraître a ; Filliettaz \& Rémery, à paraître).

\section{3.- L'accompagnement tutoral comme configuration de participation à l'interaction}

Aborder l'activité tutorale non plus seulement comme un processus abstrait et décontextualisé, mais comme un accomplissement collectif et construit par les participants dans le cours même de leur rencontre peut donner lieu à la mise en évidence de «configurations de participation». Les configurations de participation désignent pour nous des droits et des obligations que contractent mutuellement les participants à l'interaction, selon les modalités par lesquelles ils s'engagent dans les activités conjointes qui les rassemblent. Les configurations de participation résultent d'une pluralité d'ingrédients.

En premier lieu, les configurations de participation à l'interaction découlent de la nature des activités conduites par les participants et plus particulièrement de la signification que ceux-ci reconnaissent mutuellement à ces activités. Cet aspect de la participation à l'interaction a été bien cerné par Goffman (1991) et son concept de «cadre de l'expérience ». La manière dont les individus font l'expérience des réalités qu'ils rencontrent dans la vie quotidienne n'est pas immédiate et univoque mais médiatisée, filtrée, par des cadres de l'expérience qui désignent un ensemble de savoirs et de savoir-faire, culturellement construits, qui permettent d'interpréter l'expérience comme relevant d'un type particulier, et de répondre à la question «qu'est-ce qui se passe ici ?». C'est notamment en mobilisant ces cadres sociaux que les individus parviennent à interpréter la signification des situations qu'ils rencontrent et d'y ajuster leurs propres manières d'y participer. Si ces cadres jouissent, sur le plan sociétal, d'une forme de réalité partagée par les membres d'une communauté, leur convocation en situation constitue une opération à chaque fois nouvelle et le produit d'un travail d'interprétation incessant. C'est en ceci que réside, pour Goffman, le caractère à la fois complexe et «vulnérable» du cadrage de l'expérience. Parce qu' «un tas de choses différentes peuvent trouver place en même temps dans la plupart des situations » (Goffman, 1991, p. 17), les individus peuvent être amenés à convoquer une pluralité de cadres de manière simultanée. Des logiques de stratification des cadres sont également en jeu, lorsque 
par exemple des «cadres primaires » sont transposés dans des «cadres transformés », qui prennent les premiers pour modèle mais qui en altèrent les conditions de réalisation. Ainsi, le rapport des participants aux contextes de leur activité n'est pas donné une fois pour toutes mais construit par l'interaction elle-même. Selon nous, la dynamique propre aux interactions verbales et la manière d'y participer constitue une des ressources par lesquelles des contextes locaux d'activité peuvent être collectivement établis et interprétés (Duranti \& Goodwin, 1992).

En prolongement des enjeux de cadrage de l'expérience, les configurations de participation à l'interaction résultent également des traits identitaires endossés par les participants au moment de s'engager dans les activités conjointes qui les rassemblent. Toute interaction nécessite de la part des participants qu'ils s'engagent dans l'activité conjointe, qu'ils coordonnent leurs contributions respectives, adoptent des attitudes culturellement et socialement adéquates, et qu'ils endossent certains « rôles» (Goffman, 1987). Au cours des échanges, les participants doivent négocier ces actions, ces places et ces rôles, en défendre la légitimité, s'arranger pour ne commettre ni impair ni menace à son image propre ni à l'image d'autrui. Les échanges sont donc régulés par une série de normes et les contributions de chacun sont marquées par le contexte et les enjeux qui président à l'interaction. Dans cette perspective, les rôles d'action endossés par les participants procèdent d'un ajustement réciproque, pouvant faire l'objet de réorganisations multiples dans le cours même de l'interaction.

Enfin, le troisième ingrédient des configurations de participation réside pour nous dans les conditions dans lesquelles les participants à l'interaction accèdent à des ressources langagières au moment d'accomplir les activités conjointes au sein desquelles ils sont appelés à s'engager et à se coordonner. Ces conditions d'accès peuvent être précisées au moyen des concepts de «cadre participatif» ou de «formats de réception » (Goffman, 1987). Toute personne qui se trouve à un moment donné dans l'espace de co-présence d'une interaction - peut être caractérisée selon son mode d'engagement dans l'interaction. Une distinction est ainsi établie entre les destinataires ratifiés - désignés ou non par le locuteur et les destinataires non-ratifiés ou bystanders, c'est-à-dire les participants occasionnels, parmi lesquels on trouve les overhearers (ceux qui accèdent à l'interaction en étant perçus par les autres) et les eaversdroppers (ceux qui épient l'interaction à l'insu des auteurs participants). Ces distinctions présentent l'avantage d'envisager le fonctionnement d'une interaction à plusieurs et mettent en exergue les différents degrés d'implication des acteurs dans l'interaction.

Ainsi définie, dans sa triple déclinaison praxéologique, identitaire et communicationnelle, la notion de «configuration de participation » offre un outil analytique explicite et précis permettant de cerner quelques traits de la complexité des interactions tutorales en situation de travail. En particulier, la notion de configuration de participation permet d'observer comment les référentes professionnelles et les stagiaires dont elles assurent l'encadrement concilient, par les cadres de l'expérience qu'elles convoquent, les enjeux à la fois éducatifs et de formation professionnelle qui se trouvent combinés dans les situations de travail. Cette notion permet également d'observer comment, par leurs modalités d'engagement dans l'interaction, les participants endossent des rôles sociaux particuliers, tout en attribuant des rôles et des places réciproques à leurs partenaires. Enfin, la notion de configuration de participation permet de décrire, dans des situations éminemment complexes du point de vue du nombre d'individus co-présents, les « formats de réception » qui président aux interaction accomplies. Ces configurations de participation ne sont ni prétablies ni figées. Elles émergent de l'engagement des participants dans des situations de travail et de formation singulières. C'est donc à travers un travail empirique méticuleux que peuvent être mises en évidence des manières particulières, pour les référentes professionnelles, d'aménager à l'intention des stagiaires des espaces de participation à l'interaction, et réciproquement, pour les stagiaires de s'engager dans de tels espaces. 


\section{4.- Les formes et transformations dynamiques des configurations de participation dans les interactions tutorales}

Les données empiriques qui alimentent cet article sont issues d'une recherche en cours ${ }^{2}$ centrée sur la construction et la transmission des compétences professionnelles dans le champ des métiers de la petite enfance. Dans le cadre de ce programme de recherche, des observations et des enregistrements audio-vidéo d'activités de travail ont été réalisés dans trois institutions de la petite enfance de la Ville de Genève, au sein desquelles nous avons suivi plusieurs stagiaires et leur référente professionnelle durant un stage d'une durée de huit semaines effectué durant la première année de la formation professionnelle. Les institutions de la petite enfance correspondaient à des Espaces de vie enfantine relevant du secteur public, et accueillant des enfants d'âge variable, oscillant entre 6 mois et 4 ans.

\begin{tabular}{|c|c|c|c|}
\hline & Institution A & Institution B & Institution $\mathbf{C}$ \\
\hline $\begin{array}{l}\text { Groupe d'enfants } \\
\text { observé }\end{array}$ & $\begin{array}{l}16 \text { enfants âgés de } 0 \text { à } 18 \\
\text { mois }\end{array}$ & $\begin{array}{l}12 \text { enfants âgés de } 1 \text { à } 2 \\
\text { ans }\end{array}$ & $\begin{array}{l}16 \text { enfants âgés de } 3 \text { à } 4 \\
\text { ans }\end{array}$ \\
\hline $\begin{array}{l}\text { Référente } \\
\text { professionnelle (REF) }\end{array}$ & $\begin{array}{l}16 \text { ans d'expérience } \\
\text { professionnelle ; } 10 \text { ans } \\
\text { d'expérience de suivi de } \\
\text { stagiaires ; } \\
\text { Formation « sensibilisation } \\
\text { de praticien-formateur» }\end{array}$ & $\begin{array}{l}21 \text { ans d'expérience } \\
\text { professionnelle ; } 1 \text { an } \\
\text { d'expérience de suivi de } \\
\text { stagiaires }\end{array}$ & $\begin{array}{l}28 \text { ans d'expérience } \\
\text { professionnelle ; } 6 \text { ans } \\
\text { d'expérience de suivi de } \\
\text { stagiaires }\end{array}$ \\
\hline $\begin{array}{l}\text { Étudiante stagiaire } \\
\text { (STA) }\end{array}$ & $\begin{array}{l}2 \text { ans d'expérience } \\
\text { préalable en tant qu'aide } \\
\text { (groupes de } 2-4 \text { ans) }\end{array}$ & $\begin{array}{l}1 \text { an et } 8 \text { mois } \\
\text { d'expérience préalable en } \\
\text { tant que stagiaire/aide } \\
\text { (groupes de } 2-4 \text { et } 3 \text { - } 4 \\
\text { ans) }\end{array}$ & $\begin{array}{l}2 \text { ans d'expérience } \\
\text { préalable en tant qu'aide } \\
\text { (groupe } 0-1 \text { an) }\end{array}$ \\
\hline $\begin{array}{l}\text { Interactions } \\
\text { vidéoscopées entre } \\
\text { référente et étudiante en } \\
\text { situation de pratique } \\
\text { professionnelle }\end{array}$ & $\begin{array}{ll}\text { - } & \text { Activité } 1 \text { en } \mathrm{T} 1: \\
& \text { Jeux libres } \\
\text { - } & \text { Activité } 2 \text { en } \mathrm{T} 2 \text { : } \\
& \text { Transvasement de } \\
& \text { graines } \\
\text { - } & \text { Activité } 3 \text { en T3 : } \\
& \text { Parcours de motricité }\end{array}$ & $\begin{array}{ll}\text { - } & \text { Activité } 1 \text { en T1 : } \\
\text { Mini-bowling } \\
\text { - } & \text { Activité } 2 \text { en T2 : } \\
\text { Jeu des doubles } \\
\text { - } & \text { Activité } 3 \text { en T3 : } \\
\text { Transvasement de } \\
\text { graines }\end{array}$ & $\begin{array}{l}\text { - Activité } 1 \text { en T1 : } \\
\text { Peinture avec les } \\
\text { mains } \\
\text { - Activité } 2 \text { en T2 : } \\
\text { Collage de fleurs } \\
\text { - Activité } 3 \text { en T3 : } \\
\text { Jeu libre }\end{array}$ \\
\hline $\begin{array}{l}\text { Durée totale des } \\
\text { activités enregistrées }\end{array}$ & 3h. 08 min. & 4h. 24 min. & 4h. 14 min. \\
\hline
\end{tabular}

Tableau 1 : Données empiriques recueillies dans chacune des trois institutions de la petite enfance étudiées

Table 1: Empirical data collected in three early childhood education institutions

Comme l'indique le tableau ci-dessus, les observations ont porté sur des activités professionnelles et de formation se référant à des groupes d'enfants d'âges variés. Le projet était ainsi de pouvoir couvrir, dans le travail d'observation, le large spectre des profils d'enfants pris en charge au sein des espaces de vie enfantine. Au sein de chacune des trois institutions, un binôme formé d'une étudiante stagiaire et de sa référente professionnelle a été observé. Dans le contexte genevois de la formation professionnelle des éducatrices de l'enfance, la situation de stage, dite «pratique professionnelle accompagnée », fait l'objet de

2 Cette recherche est soutenue par le Fond National Suisse pour la recherche scientifique (No CRSII1-136291) pour la période 2012-2014. Elle s'inscrit dans le cadre du programme Sinergia « Young people's interactional competences in institutional practices : between school and the workplace » (IC-You) et du sous-projet intitulé « Building interactional competences in Vocational Education and Training (VET) programs: the case of early childhood educators ». Participent à ce sous-projet, outre les trois signataires de cet article, Vassiliki Markaki, Stefano Losa, Isabelle Durand et Marianne Zogmal. 
prescriptions émanant de l'école professionnelle supérieure. Selon ces prescriptions, il est attendu des référentes professionnelles qu'elles assurent aux étudiantes un accompagnement pédagogique leur permettant d'atteindre les objectifs du stage, qu'elles explicitent à leur intention les gestes du métier et qu'elles les aident à identifier leurs potentialités et leurs difficultés. Ceci suppose un travail au quotidien en proximité des stagiaires et la conduite régulière d'entretiens pédagogiques permettant d'engager un processus réflexif à propos des activités réalisées.

Les référentes sélectionnées pour ces observations avaient toutes pour caractéristique le fait a) d'être des éducatrices diplômées, b) disposant d'une expérience professionnelle substantielle et reconnue, et c) ayant encadré des stagiaires depuis au moins une année. Les étudiantes stagiaires disposaient toutes d'une expérience professionnelle préalable en lien avec des contextes éducatifs. Elles ont été observées en fin de première année de formation, à l'occasion d'un stage dit «d'observation et d'expérimentation» de huit semaines, dont l'objectif était de mettre en œuvre auprès des enfants des activités sous-tendues par des objectifs pédagogiques explicites.

Dans chacune des trois institutions, les observations filmées ont été planifiées à trois moments distincts du stage : en début (T1), en milieu (T2) et en fin de stage (T3). Lors de chaque observation, une activité éducative prise en charge par le binôme constitué de la stagiaire et sa référente a été filmée, précédée et suivie des temps de transition. Certaines de ces activités étaient «libres» et consistaient à laisser les enfants explorer librement l'environnement ludique qui leur était proposé ; d'autres étaient en revanche conçues comme «structurées » et prévoyaient des modalités de participation plus contraignantes pour les enfants (ex. parcours de psychomotricité, jeu de bowling, jeux de cartes, bricolages, etc.).

En lien avec ces données d'observation filmées, trois sortes de données complémentaires ont été recueillies dans le cadre de la présente recherche : a) des documents méthodologiques produits par les stagiaires au moment de planifier les activités éducatives réalisées, b) des entretiens pédagogiques réalisés entre les stagiaires et leur référente professionnelle, c) des entretiens de recherche, réalisés avec chacun des binômes en fin de stage.

Ce matériau empirique a fait l'objet de plusieurs analyses, dont certaines sont encore en cours et d'autres ont fait l'objet de publications (Filliettaz, à paraître b, à paraître c ; Filliettaz \& Rémery, à paraître ; Markaki \& Rémery, 2013 ; Zogmal, Losa \& Filliettaz, 2013). Pour les besoins de cet article, nous nous centrons principalement sur l'analyse des données interactionnelles issues des enregistrements audio-vidéo d'activités. L'analyse de ces données situées nous permet d'observer comment se construisent et se négocient des configurations de participation dans les situations de formation à la pratique professionnelle. En particulier, ces données illustrent comment les référentes professionnelles aménagent pour les stagiaires des opportunités de participation à des activités éducatives, et réciproquement comment les étudiantes en formation investissent ces opportunités de participation pour expérimenter, sous différentes facettes, les compétences professionnelles visées dans leur parcours de formation.

Pour dégager de telles configurations, les données ont d'abord fait l'objet d'une description synoptique dans le cadre d'un travail de préanalyse et de transcription effectuée à l'aide du logiciel Transana Multi-User (http://www.transana.org/). La démarche de préanalyse et de transcription consiste à décrire de manière détaillée le contenu des enregistrements disponibles et le déroulement temporel général des activités observables dans ces enregistrements. Cette démarche préparatoire vise à repérer, dans les données brutes recueillies, des séquences pertinentes pouvant donner lieu à une analyse approfondie. Sur la base de ce travail de préanalyse, une collection de séquences a été réalisée par les chercheurs, fondée sur des propriétés variables des ingrédients de l'organisation de l'interaction (voir 3.). Cette collection a permis de repérer des régularités et de mettre en évidence trois formats d'accompagnement tutoral que nous avons désignés comme a) de 
l'observation, b) de la co-animation, et c) de la monstration. Chacun de ces formats donne à voir les types d'interactions qui y prennent place et la nature de la relation qui s'instaure à un moment donné d'une activité éducative entre la référente et la stagiaire. Ces configurations nous renseignent également sur la façon dont les stagiaires s'alignent ou non à ces formes de participation qui leur sont proposées. Dans les paragraphes qui suivent, nous précisons et illustrons par de courts extraits de données ces trois configurations de participation et les dynamiques par lesquelles elles se mettent en place et se transforment dans l'interaction. Ces extraits ont été sélectionnés pour leur caractère emblématique du format d'accompagnement tutoral dont ils relèvent.

\section{1.- La configuration d'observation}

Un premier format d'accompagnement observé dans les institutions visitées peut être désigné comme une configuration d'observation. Dans cette configuration, la référente professionnelle place la stagiaire dans une situation de «mise au travail faiblement assistée », pour reprendre une des catégories proposées par Kunégel (2011). Elle confie à la stagiaire la responsabilité de l'animation de l'activité éducative et se tient ostensiblement en retrait de l'animation, endossant une posture d'observation. Cette observation présente alors un caractère évaluatif, l'activité réalisée par la stagiaire donnant lieu à diverses appréciations formulées soit en cours d'animation, soit de manière consécutive, à l'occasion des entretiens pédagogiques qui ponctuent les étapes du stage.

Pour illustrer la manière dont se met en place et se transforme ce type de configurations de participation, nous proposons un premier exemple, en lien avec le binôme référente-stagiaire observé au sein de l'institution C en début de stage (T1). La stagiaire (STA) a prévu d'animer une activité de peinture avec un groupe de trois enfants âgés de 4 ans (Activité 1). La spécificité de cette activité réside dans le fait que les enfants sont invités à peindre un arbre directement avec leurs mains. À cette fin, de grandes feuilles de papier ont été placées sur un mur et des assiettes contenant de la peinture de différentes couleurs ont été préparées. Au moment de débuter cette activité, la stagiaire rassemble les trois enfants sur un banc. Elle commence par leur lire un livre dont les illustrations représentent des tableaux peints avec des mains. Au terme de la lecture, elle annonce aux enfants l'activité prévue :

Extrait $1:$ « faire un arbre avec les mains » $(33: 30-34: 23)^{3}$

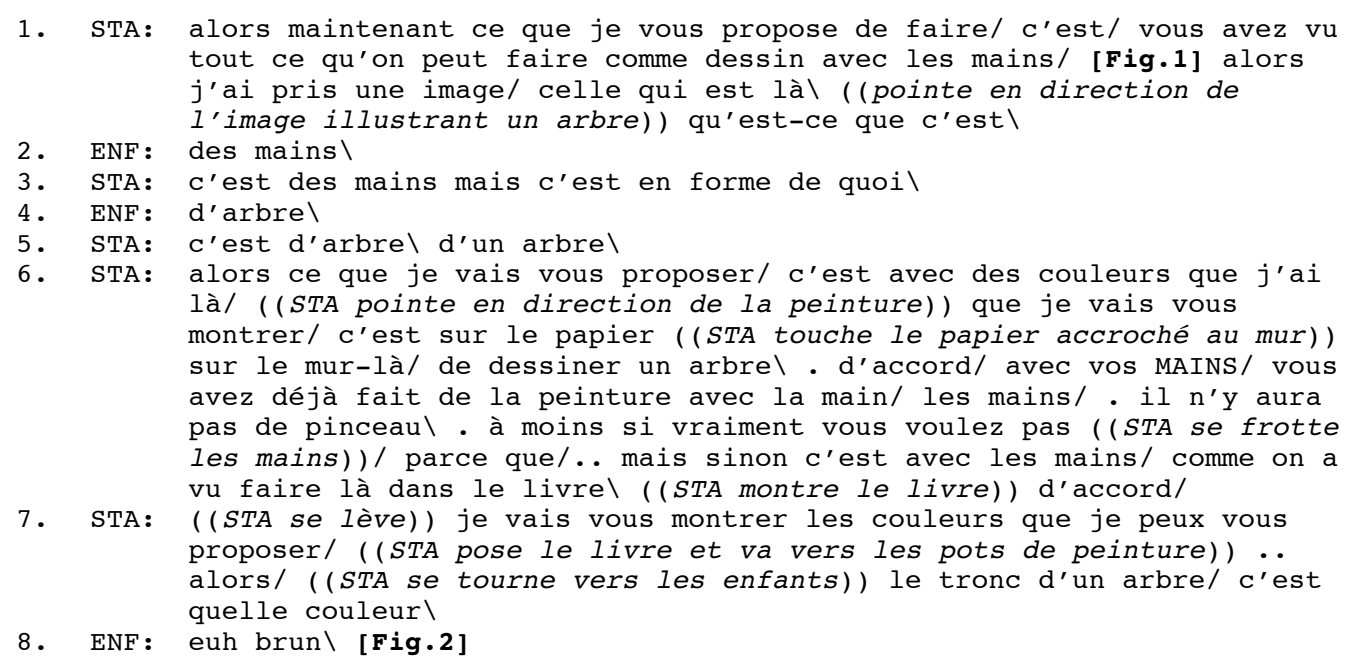

3 Les conventions de transcription sont indiquées en Annexes, en fin d'article. 


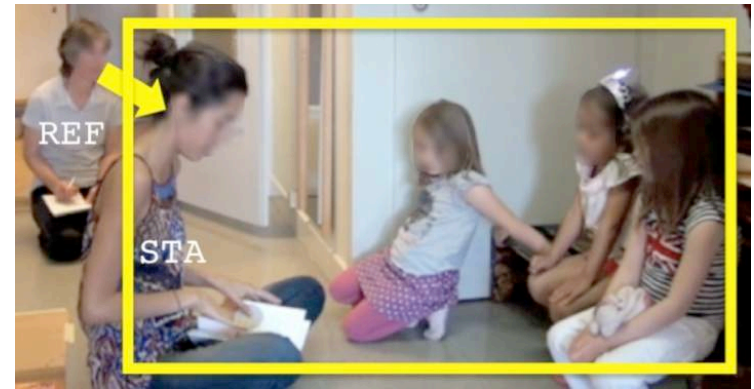

Figure 1 - 00:33:37

STA termine la lecture du livre ; REF regarde en direction de STA

\section{Figure 1: STA finishes to read the book; REF looks towards STA}

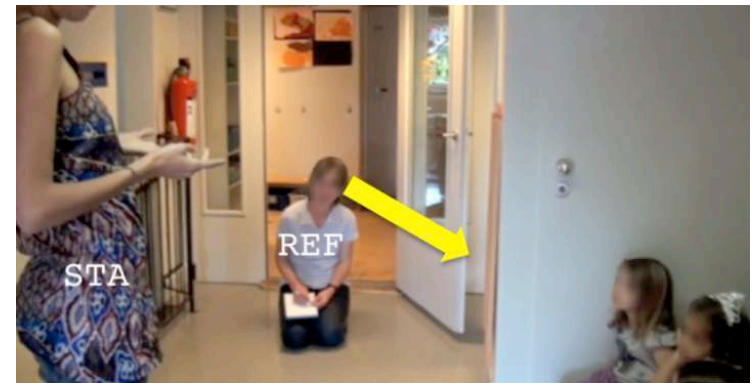

Figure 2 - 00:34:23

STA donne les consignes ;

REF regarde en direction des enfants.

\section{Figure 2: STA gives instructions to the group of children; \\ REF looks towards the children}

Dans l'extrait 1, retranscrit ci-dessus, on observe comment la stagiaire investit un espace d'autonomie aménagé par la référente auprès des enfants. C'est elle qui prend en charge l'animation de l'activité peinture et qui en accomplit séquentiellement les étapes constitutives. Arrivée au terme de la lecture du livre, elle demande aux enfants d'énoncer les caractéristiques des illustrations tirées du livre. Elle engage pour ce faire des formes interrogatives d'étayage («qu'est-ce que ç'est», ligne 1 ; «mais c'est en forme de quoi », ligne 3) auxquelles les enfants s'alignent immédiatement («des mains», ligne 2; «d'arbre », ligne 3). Une fois ces spécificités repérées, la stagiaire procède à l'annonce de l'activité prévue («je vais vous proposer [...] sur le mur-là de dessiner un arbre avec vos mains », ligne 6) et présente successivement les ingrédients matériels qui seront mobilisés. À cette fin, la stagiaire pointe les différents éléments dans l'espace par son orientation corporelle ou diverses conduites gestuelles.

La référente (REF) quant à elle n'intervient pas explicitement dans l'animation de cette activité. Comme indiqué sur les Figures 2 et 3, elle se tient en retrait du foyer d'interaction formé par la stagiaire et les enfants. Son regard porte tantôt sur la stagiaire (Figure 1), tantôt sur les enfants (Figure 2), notamment lorsqu'une réponse est attendue de leur part ( « le tronc d'un arbre c'est quelle couleur/», ligne 8). Mais à ce stade, la référente n'accède ni à une position de locutrice ni à celle de destinataire directe des propos échangés. Elle constitue, dans les termes de Goffman (1987), un «témoin ratifié » de l'interaction. Les modalités locales de participation qui résultent d'une telle configuration présentent dès lors un caractère à la fois complexe et stratifié. En effet, l'activité dans laquelle la référente s'engage publiquement ne relève pas du cadre primaire de l'animation éducative auprès des enfants mais d'une autre activité, qui prend la première comme objet d'observation, et qu'on pourrait désigner comme le cadre de la formation. On retrouve ici un cas particulier de ce que Goffman (1991) désigne comme une «modalisation», c'est-à-dire un ensemble de conventions en vertu desquelles une activité est transformée par les conditions de réalisation d'une seconde activité, qui prend la première pour objet, et qui en modifie la signification et les conditions de réalisation. Cette stratification des cadres de l'activité éducative et de l'activité formative affecte en profondeur l'identité endossée par la référente dans la situation. La référente endosse en effet publiquement à ce moment de l'activité un rôle de «formatrice» et non pas «d'éducatrice», comme en attestent notamment les objets matériels qu'elle manipule et l'usage qu'elle en produit (ex. la prise de notes sur un bloc de feuilles).

Cette stratification du contexte local ne demeure cependant pas uniformément étanche et continue. À plusieurs reprises dans la suite de cette interaction, des écarts à une position de retrait sont observables chez la référente. Ces écarts engagent de fréquentes reconfigurations des modalités de participation de la stagiaire. Ce type de transformations locales de la 
configuration de participation peut être observé dans l'extrait 2, qui intervient quelques minutes plus tard, au moment où la référente se penche en direction de la stagiaire pour lui faire une demande :

Extrait $2:$ «essuie voir les traces qui sont par terre » (45:18-45:43)

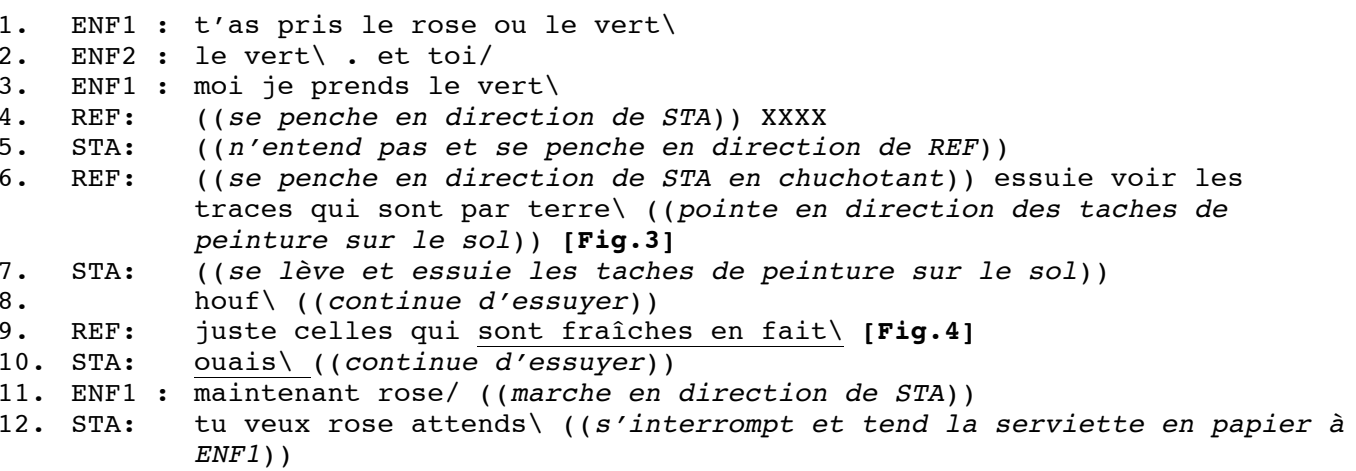

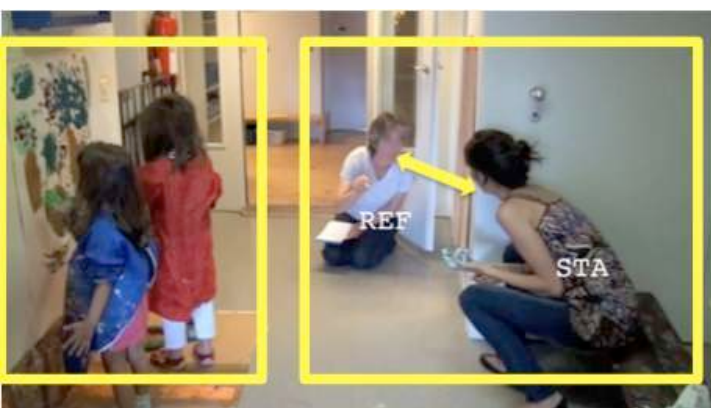

Figure 3-00:45:24

REF attire l'attention de STA sur la présence de taches de peinture sur le sol

\section{Figure 3: REF draws STA's attention to paints spots on the ground}

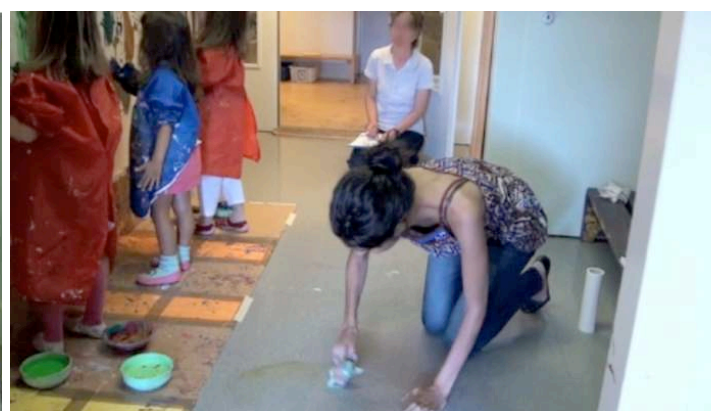

Figure 4-00:45:38

STA nettoie la tache de peinture sous le regard et les conseils de REF

Figure3: STA cleans fresh paint spots on the
ground

$\mathrm{Au}$ moment où débute l'extrait 2, les trois fillettes sont activement engagées dans la réalisation de leurs peintures. Elles interagissent à propos de leurs choix de couleurs (ligne 13), sous le regard de la stagiaire. Pendant ce temps, la référente observe que les enfants transportent de la peinture fraîche sous leurs pantoufles et laissent des traces sur le sol. Cet événement, non anticipé par la stagiaire dans la planification de son activité, agit sur les modalités de participation à l'interaction de la référente qui va reconfigurer la posture d'observatrice et de témoin ratifié qu'elle endossait dans l'extrait (1). La référente se penche alors en direction de la stagiaire et lui chuchote des instructions (ligne 4) puis pointe à nouveau en direction du sol en énonçant des directives à son attention : « essuie voir les traces qui sont par terre» (ligne 6). Par ces interventions, qui prennent des formes sémiotiques diverses, la référente fait intrusion en quelque sorte dans le cadre de l'activité éducative dont elle s'était retirée précédemment. Elle rend visible par cette forme d'interpellation auprès de la stagiaire le cadre de la formation qu'elle projette sur la situation. Pour le dire autrement, elle exerce localement une forme de guidage de l'activité éducative, en mettant à la disposition de la stagiaire des ressources ponctuelles lui permettant de gérer une situation imprévue. De manière intéressante, ces ressources ne sont pas portées à l'attention des enfants mais placent la stagiaire comme seule destinatrice de l'interaction. On pourra noter à ce propos que les instructions de la référente sont produites sur le registre prosodique du chuchotement, à un moment où les enfants sont engagés dans l'acte de peindre et n'orientent temporairement pas leur attention en direction de la stagiaire. Dans ce contexte, le cadre de la formation se déploie «en coulisse » d'une activité éducative dans laquelle la référente montre qu'elle n'agit pas au titre de participant ratifié (voir Figure 3). 
La stagiaire s'aligne à ce changement de participation et satisfait immédiatement à cet acte directif en se déplaçant et en frottant le sol avec du papier. Elle reprend ensuite rapidement le contrôle de l'activité et se réoriente dans le cadre primaire de son activité éducative au moment où les enfants la sollicitent à nouveau concernant leur choix de peinture (« tu veux du rose attends », ligne 12). La référente reconfigure alors sa participation et revient à sa posture d'observatrice, non sans noter sur son carnet des commentaires qu'elle restituera ultérieurement à la stagiaire au moment de l'entretien pédagogique

Dans ce deuxième extrait, on relève donc comment la référente quitte ponctuellement un format d'observation de l'activité pour orienter l'attention de la stagiaire sur des propriétés de la situation qui apparaissent comme non anticipées et non perçues par la stagiaire. C'est là peut-être que résident les spécificités des dynamiques de participation que sous-tend la configuration d'observation. Sans pour autant s'effacer complètement, la référente propose des interventions brèves et ciblées qui répondent à des «événements » imprévus et permettent de les surmonter. Du côté de la stagiaire, des activités éducatives mises en œuvre peuvent ainsi être expérimentées à proximité de ressources disponibles, en cas de besoin.

\section{2.- La configuration de co-animation}

Il existe cependant des configurations de participation de l'activité tutorale qui prennent des formes distinctes de celles que nous venons de décrire, et qui peuvent être désignées comme de la co-animation. La configuration de co-animation désigne des formats de participation dans lesquels la référente et la stagiaire se distribuent des tâches distinctes et complémentaires dans une activité éducative qu'elles réalisent conjointement. Dans cette configuration de participation, le cadre de la formation ne se situe pas à l'extérieur du cadre primaire d'une activité éducative prise pour objet d'observation et d'évaluation. Il s'exprime au cœur même de la conduite de l'activité engagée auprès des enfants.

Afin d'illustrer diverses formes de manifestation de cette configuration de co-animation, nous proposons de nous reporter à des situations d'interaction recueillie à propos d'un binôme distinct, observé au sein de l'institution B en début de stage (T1). Suite à une situation de jeu libre dans laquelle plusieurs adultes accompagnent les enfants dans l'exploration de leur environnement, un groupe restreint transite vers une salle de motricité aménagée au préalable par la stagiaire. Ce groupe est composé de la stagiaire (STA), de la référente (REF) et de quelques enfants âgés de 3 ans environ (ELI, BEN, ALI, etc.). La stagiaire propose de conduire une activité, appelée ici «mini-bowling » (Activité 1). Le jeu consiste à faire rouler des balles en direction de bouteilles en plastique pour les faire tomber. Sans pour autant prendre la main sur l'activité, la référente propose diverses formes d'interventions tutorales qui, à plusieurs égards, se distinguent de celles relevées précédemment, et qui méritent d'être mises en évidence. Dans l'extrait 3, nous observons pour commencer comment la stagiaire présente l'activité "mini-bowling» au groupe d'enfants et comment elle formule des consignes à leur intention :

Extrait 3 : « écoute Élie ! » (33:33-34:22)

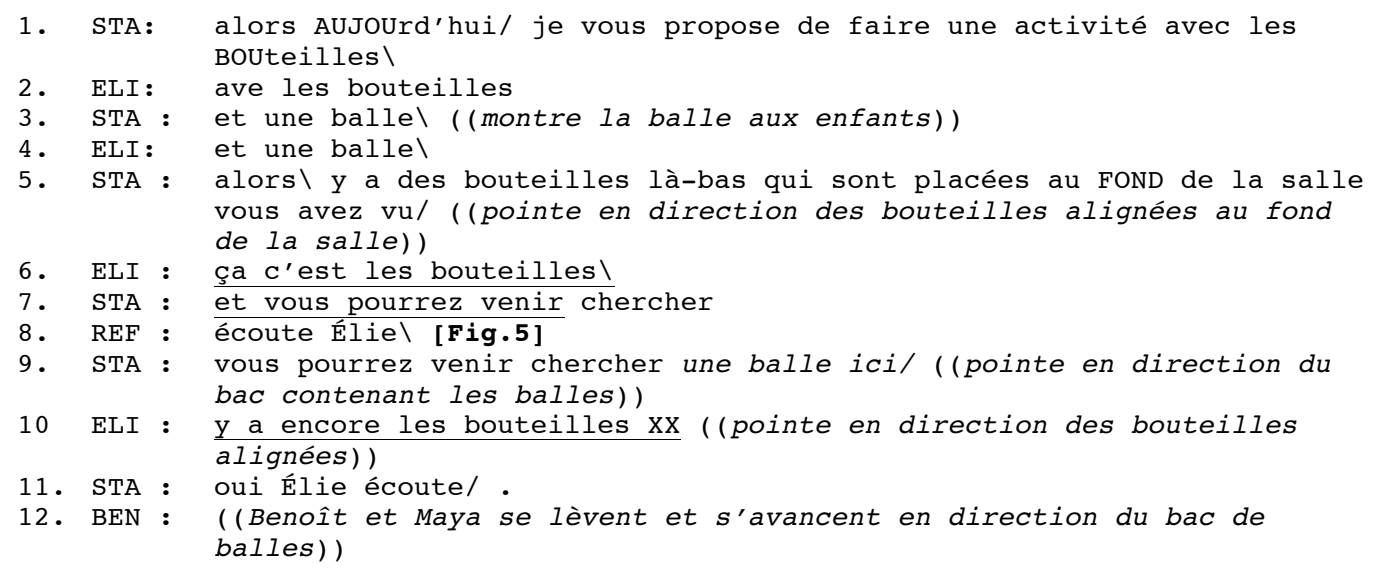




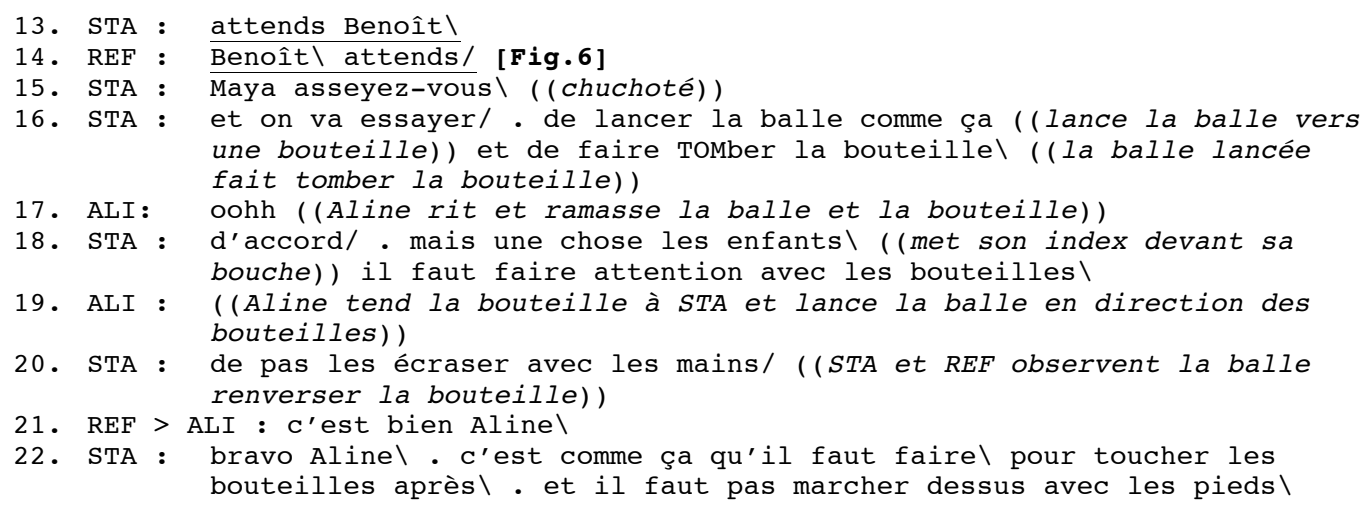

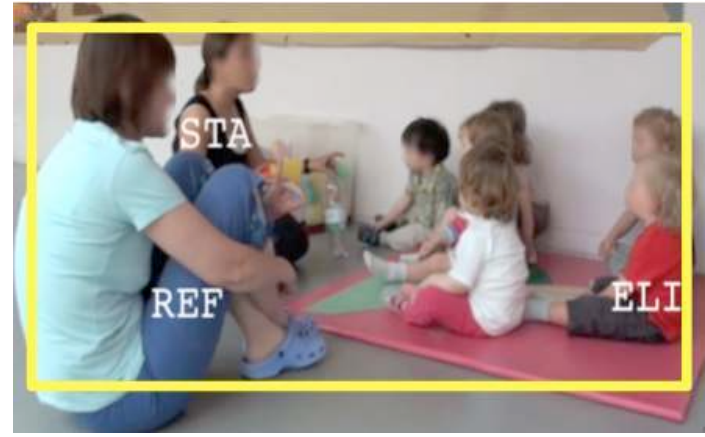

Figure 5 - 00:33:50

STA donne les consignes aux enfants ; REF intervient auprès d'ELI

\section{Figure 5: STA gives instructions to the children;}

\section{REF engages with ELI}

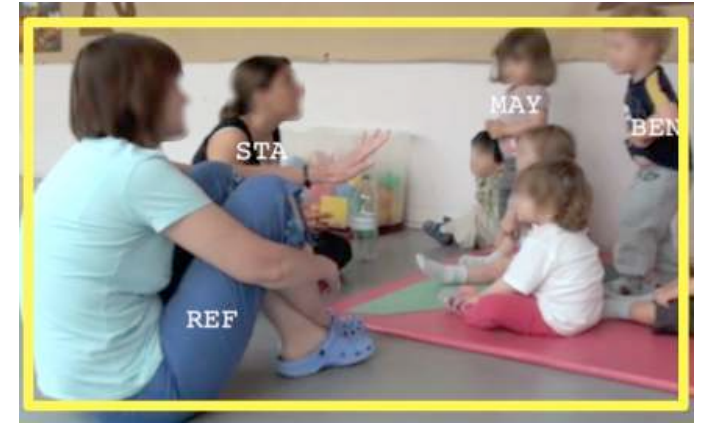

Figure 6 - 00:33:56

REF et STA demandent à Maya et Benoît d'attendre

Figure 6: REF and STA ask MAY and BEN to sit down and wait for the game to start

Pour présenter l'activité, la stagiaire et la référente ont rassemblé les enfants sur un tapis en mousse situé devant un bac contenant des balles. Des bouteilles de plastique ont été disposées au fond de la salle, dans le dos de la stagiaire. La stagiaire et la référente adoptent une position corporelle similaire. Elles sont toutes deux assises sur le sol et font face aux enfants (voir Figures 5 et 6). C'est pourtant bien la stagiaire qui prend en charge l'animation. Elle commence par présenter aux enfants les objets qui seront utilisés dans l'activité «minibowling», successivement les bouteilles («je vous propose de faire une activité avec les bouteilles », ligne 1) et les balles (« et une balle», ligne 3). Elle poursuit par une démonstration de l'activité consistant à lancer une balle en direction d'une bouteille (« et on va essayer de lancer la balle comme ça et de faire tomber la bouteille », ligne 16). Enfin, la stagiaire énonce à l'intention des enfants des consignes concernant les conditions de réalisation de l'activité ( «mais une chose les enfants il faut faire attention avec les bouteilles de ne pas les écraser avec les mains », ligne 18-20).

Les enfants s'engagent activement et selon des modalités variées dans cette activité de consigne. Élie, assis à l'arrière du groupe (voir Figure 5), observe attentivement la stagiaire et reformule littéralement ses propos («ave les bouteilles», ligne 2 ; « et une balle», ligne 4 ; «ça c'est les bouteilles », ligne 6). Benoît et Maya anticipent prématurément le démarrage de l'activité, se lèvent et se dirigent vers le bac pour se saisir de balles (voir Figure 6). Enfin, Aline, assise en face de la stagiaire, s'approprie la balle utilisée pour la démonstration et la lance en direction des bouteilles avant même la fin de la consigne (ligne 19). C'est d'ailleurs systématiquement à propos de ces formes d'engagement des enfants que la référente intervient dans l'animation de l'activité. Élie et Benoît sont interpellés à plusieurs reprises, avant ou après des régulations opérées par la stagiaire («écoute Élie », ligne 8 ; «Benoît attends », ligne 14). Enfin, la référente ratifie la tentative d'Aline consistant à lancer la balle en direction des bouteilles (« c'est bien Aline », ligne 21).

Ces interventions de la référente dans l'activité façonnent une configuration de participation 
particulière dans laquelle le cadrage tutoral s'exprime au sein même de l'activité éducative conduite auprès des enfants. La référente n'observe pas en tant qu'évaluatrice externe une activité conduite par la stagiaire mais elle prend une part active à l'animation du jeu, notamment en centrant ses interventions sur des actes de régulation du comportement des enfants. Dans ces régulations, elle sélectionne les enfants comme des destinataires directs et se positionne elle-même comme un participant ratifié de l'activité éducative en cours d'accomplissement. La stagiaire s'aligne visiblement aux interventions adressées par la référente aux enfants, notamment par des phénomènes de reprise en écho des régulations énoncées. Elle reformule par exemple l'injonction adressée à Élie («oui Élie écoute », ligne 11), demande également à Benoît et Maya d'attendre («attends Benoît [...] Maya asseyez-vous », ligne 13-15) et élabore la validation de la tentative d'Aline («bravo Aline c'est comme ça qu'il faut faire pour toucher les bouteilles après », ligne 22).

La configuration de co-animation repose parfois sur des formes d'engagements plus clairement complémentaires entre la stagiaire et la référente, comme par exemple dans l'extrait 4, qui retrace le démarrage effectif de l'activité «mini-bowling »:

Extrait $4:$ : va chercher une balle » (34:31-34:55)

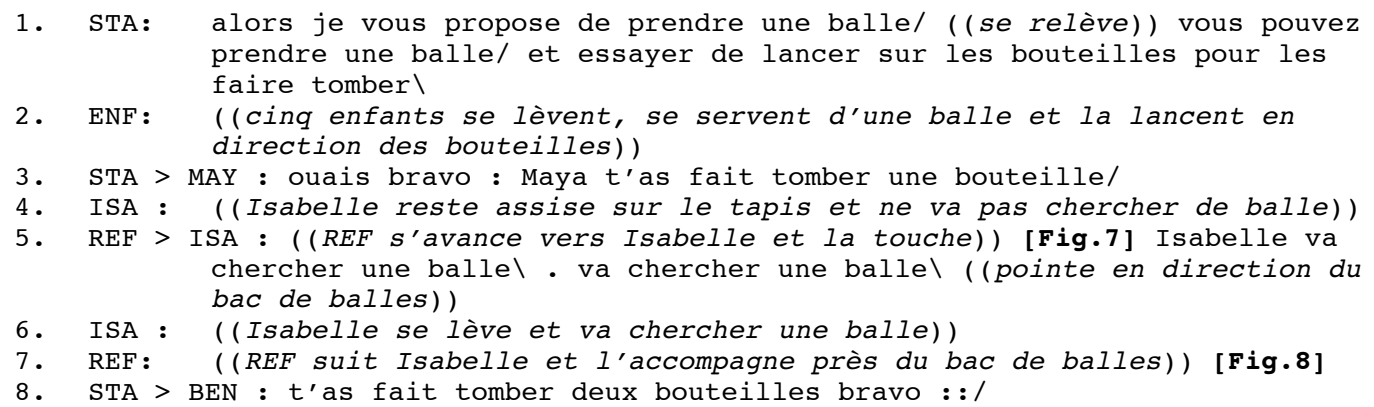

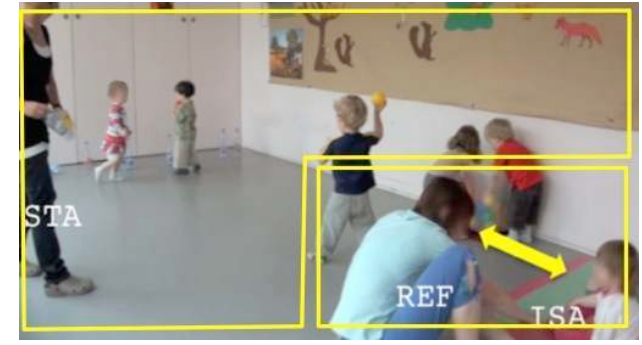

Figure 7 - 00:34:44

STA initie l'activité pendant que REF invite ISA à prendre part au jeu

Figure 7: STA initiates the bowling game while REF brings ISA back into the group

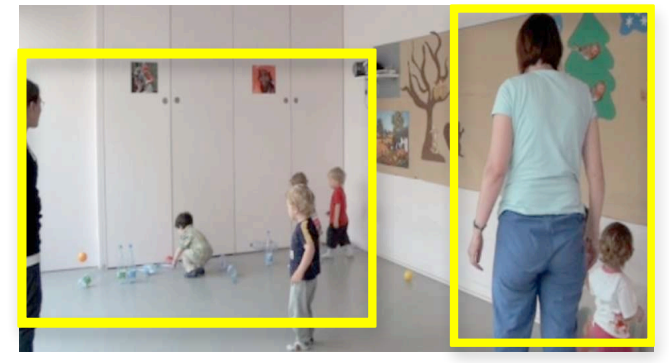

Figure 8-00:34:54

REF accompagne ISA vers le bac de balles pendant que STA joue avec le groupe d'enfants

Figure 8: REF guides ISA to the box containing the bowls while STA plays with the group

Arrivée au terme de ses consignes, la stagiaire annonce aux enfants qu'ils peuvent se mettre en activité («alors je vous propose de prendre une balle vous pouvez prendre une balle et essayer de lancer sur les bouteilles pour les faire tomber», ligne 1). Un groupe de cinq enfants se lève alors immédiatement et commence à lancer les balles en direction des bouteilles. Une fillette, Isabelle, reste cependant en retrait et ne s'engage pas dans l'activité proposée. Dans cette configuration de participation, la stagiaire et la référente s'engagent de manière distincte dans la situation de jeu. La stagiaire s'oriente vers les enfants engagés dans le jeu, notamment en évaluant positivement leurs tentatives ( tomber une bouteille», ligne 3 ; «t'as fait tomber deux bouteilles bravo », ligne 8). La référente quant à elle s'oriente principalement en direction d'Isabelle et s'emploie à l'engager dans l'activité, d'abord par des injonctions («Isabelle va chercher une balle », ligne 5) puis en l'accompagnant en direction du bac contenant les balles (voir Figure 8). 
Comme permettent de le souligner les Figures 7 et 8 , la configuration de participation qui résulte de cette séquence se structure autour de «régions ${ }^{4}$ distinctes de l'environnement. Un premier foyer d'activité est animé par la stagiaire et porte sur l'accomplissement collectif du jeu ; un second foyer d'activité est animé par la référente et porte sur l'accompagnement individuel d'une des participantes. Mais chacun de ces foyers relève bien d'un cadrage lié à la conduite de l'activité éducative auprès des enfants. C'est dans ce sens que s'exprime ici aussi une forme complémentaire de co-animation.

Il apparaît donc dans ces différentes vignettes que la configuration de co-animation peut prendre des formes variées, allant de la ratification par la stagiaire d'interventions initiées par la référente à des formes explicites de coordination, en passant par des modalités d'engagements distinctes et complémentaires auprès des enfants. Ce qui spécifie la coanimation et qui notamment la distingue de l'observation, c'est l'établissement d'un engagement convergent de la référente et de la stagiaire en direction d'un cadre éducatif centré sur les enfants. C'est en ceci que cette forme d'accompagnement tutoral peut sembler moins visible que d'autres. Rarement ostensible et explicite, la co-animation peut se confondre avec l'activité éducative elle-même et présenter une forme d'invisibilité sur le plan des enjeux de formation qui président aux situations observées. Pourtant, ce que les données et leur analyse montrent bien, c'est le caractère potentiellement étayant de ces formes d'intervention de la référente, qui, tout en s'adressant aux enfants dans le cadre de l'animation d'une activité éducative, met en visibilité à destination de la stagiaire des ressources à mobiliser à l'occasion par exemple de régulations ou de relances. Par ses interventions directives, ses prises en charges complémentaires ou ses anticipations, la référente contribue à atténuer la complexité des situations éducatives que rencontre la stagiaire au moment de s'engager dans son activité. C'est dans ce sens que le format de la co-animation constitue une forme d'aménagement des conditions d'engagement de la stagiaire et qu'il peut être, en tant que tel, perçu comme une ressource de l'activité tutorale.

\section{3.- La configuration de monstration}

Il existe enfin des configurations de participation dans lesquelles la mise à disposition de ressources prend des formes plus explicites. Tel est par exemple le cas du format de la monstration, déjà repéré par Kunégel (2011) dans un contexte de formation différent. La configuration de monstration désigne pour nous des situations proches de la co-animation, mais dans lesquelles l'activité éducative conduite par la référente auprès des enfants présente, à des degrés divers, un caractère ostensible à l'attention de la stagiaire. La stagiaire est alors elle-même placée dans une position d'observatrice et invitée à repérer des traits significatifs et reproductibles d'une activité exemplaire réalisée par la référente. Comme les autres configurations de participation relevées ci-dessus, la monstration procède d'un engagement réciproque et complémentaire de la référente et de la stagiaire. La question se pose dès lors d'observer comment la stagiaire repère ces traits ostensibles de l'activité de la référente et surtout quels usages elle fait de ces traits dans sa propre activité.

Pour alimenter ce questionnement, nous proposons ci-dessous de nous référer à un binôme, observé dans notre démarche de recherche au sein de l'institution A en fin de stage (T3). L'activité éducative dont nous proposons d'analyser quelques extraits consiste en un parcours de psychomotricité conçu et aménagé par la stagiaire en question à l'intention d'un groupe d'enfants âgés de 2 ans environ (Activité 3). L'espace aménagé par la stagiaire est organisé en quatre zones d'activités délimitées par des modules de psychomotricité, incluant notamment un toboggan, un tunnel en mousse, un cylindre et des ballons. Les enfants explorent à leur guise cet environnement, sous la conduite de la stagiaire et en présence de sa référente. Nous proposons de montrer ci-dessous comment, à certains moments, la référente

4 Nous entendons ici le terme « région » dans un sens proche de celui que lui a donné Goffman (1973, p. 105), soit « un lieu borné par des obstacles à la perception». 
enrichit le format de la co-animation par des conduites ostensibles adressées à la stagiaire, et comment la stagiaire s'approprie progressivement ces conduites ostensibles pour « reprendre la main » sur son activité.

Au moment où débute l'extrait 5 , dix minutes se sont déjà écoulées depuis l'entrée des enfants dans la salle de psychomotricité. Les enfants ont exploré spontanément certains modules comme le tunnel et le toboggan mais en ont ignoré d'autres. La référente (REF) et la stagiaire (STA) sont positionnées dans des régions distinctes de l'environnement. La stagiaire joue avec Dominique (DOM), près de la porte («vas-y », ligne 1), pendant que la référente aide un groupe d'enfants à monter sur le toboggan. À ce moment, Ludivine (LUD), une des fillettes du groupe, s'approche du module cylindrique posé horizontalement sur le sol et qu'aucun enfant n'a encore utilisé. La transcription ci-dessous montre comment la référente exploite cette opportunité que constitue l'intérêt de Ludivine pour le cylindre et comment elle reconfigure à cette occasion les conditions de participation non seulement du groupe d'enfants mais encore de la stagiaire :

Extrait $5:$ : on peut le mettre comme ça » $(36: 10-37: 55)$

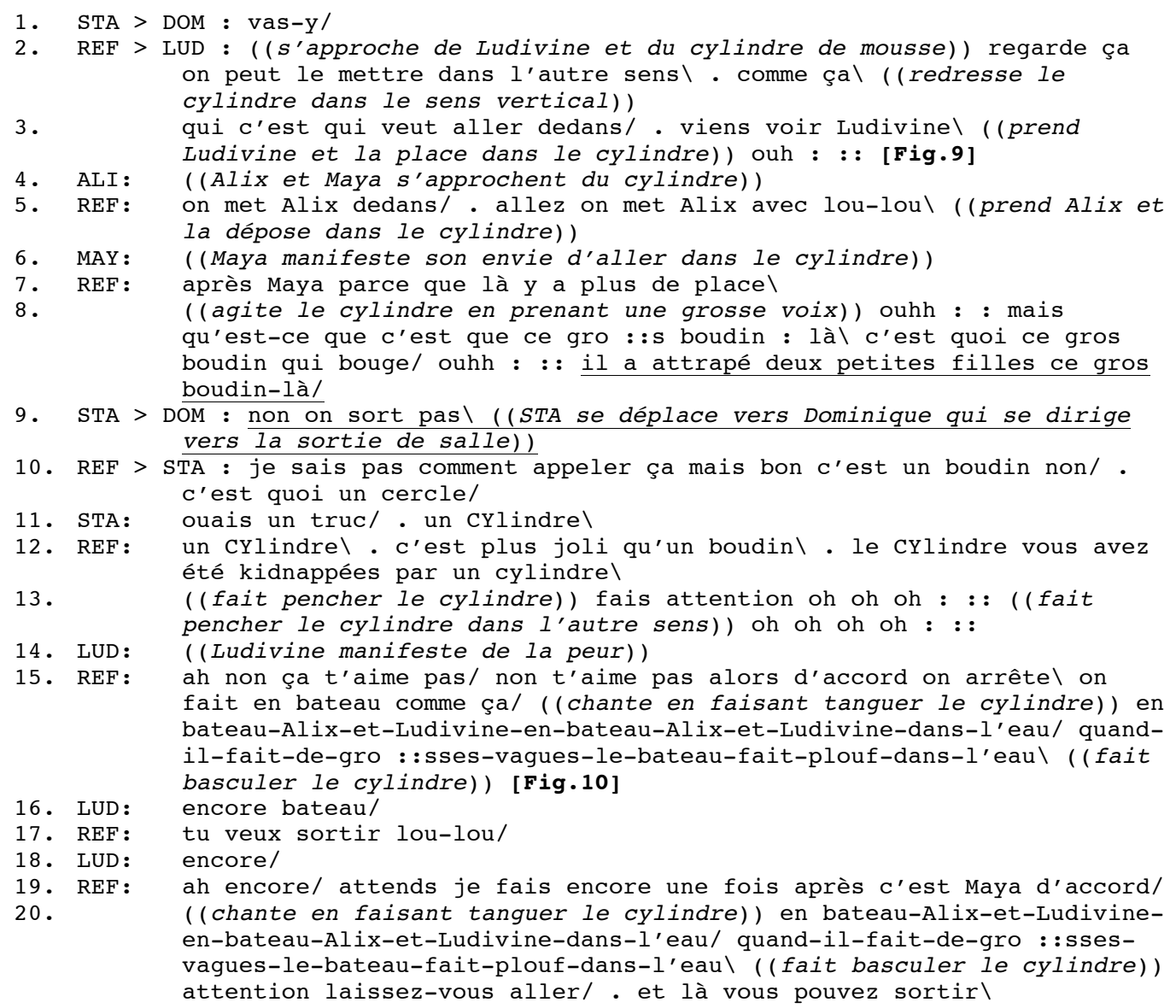




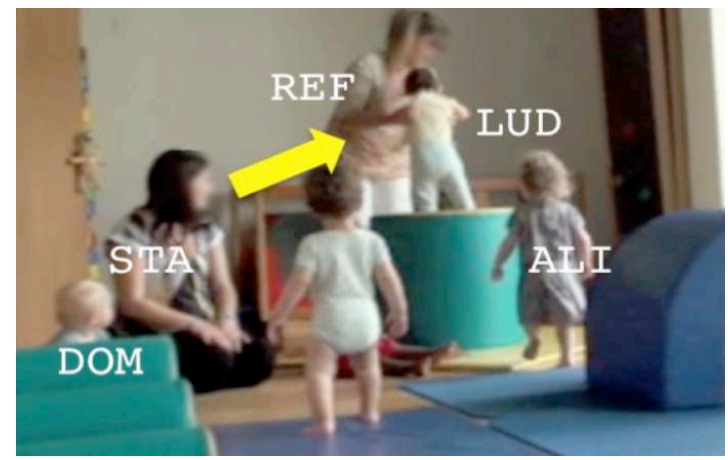

Figure $9: 00: 36: 22$

Sous le regard de STA, REF place le cylindre en position verticale et place LUD à l'intérieur

\section{Figure 9: STA observes how REF places $L U D$ inside the cylinder}

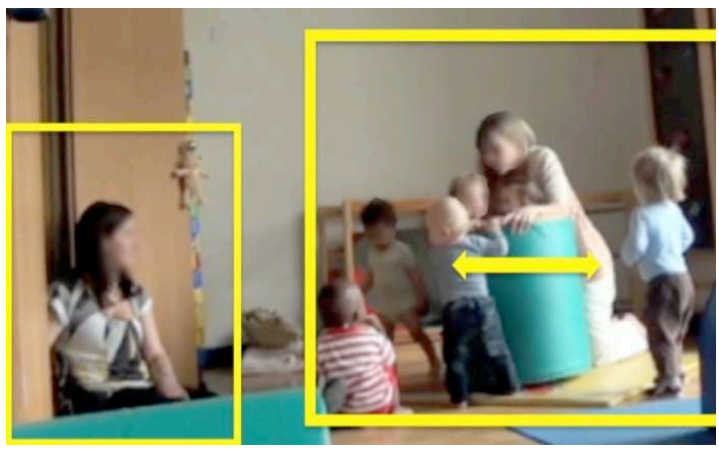

Figure $10: 00: 37: 37$

REF fait tanguer le cylindre en chantant la chanson du bateau

\section{Figure 10: REF rocks the cylinder while singing the boat song}

La reconfiguration de la participation initiée par la référente procède par étapes successives. La référente commence par se déplacer et se rapprocher de Ludivine en verbalisant le fait que le cylindre peut être placé dans un sens différent (« regarde ça on peut le mettre dans l'autre sens », ligne 2). Elle le positionne alors elle-même sur un axe vertical, ce qui offre de nouvelles possibilités de jeux aux enfants. La référente va alors proposer de placer Ludivine au centre du cylindre (« qui c'est qui veut aller dedans viens voir Ludivine », ligne 3), ce qui attire immédiatement l'attention des autres enfants et de la stagiaire en direction de ce nouveau foyer d'activité (voir Figure 9). Après avoir placé Alix au centre du cylindre, la référente anime successivement plusieurs brèves activités. Elle commence par faire tanguer le cylindre en prenant une grosse voix (ligne 8), avant de reproduire en l'adaptant la chanson «en bateau » (ligne 15-19). A l'occasion de ces animations, la référente interpelle également la stagiaire au sujet de la désignation du «boudin »: «je sais pas comment appeler ça mais bon c'est un boudin non c'est quoi un cercle/ » (ligne 10). Les deux éducatrices se mettent alors d'accord pour désigner l'objet comme un «cylindre » (« ouais un truc un cylindre », ligne 11 ; « un cylindre c'est plus joli qu'un boudin », ligne 12).

Ce qui retient notre attention dans cette séquence, c'est le rôle configurant qu'endosse la référente à ce moment d'une activité placée sous la responsabilité déclarée de la stagiaire. Loin de s'effacer selon les modalités du format de l'observation ou de se cantonner à un rôle de co-animation, la référente prend à ce moment la main sur l'activité. Elle relance l'intérêt des enfants pour une région de l'environnement encore inexplorée et réoriente en profondeur l'engagement des enfants comme de la stagiaire en direction du cylindre. Même Dominique, jusqu'ici engagé dans un jeu avec la stagiaire, se déplace et rejoint la référente autour du cylindre (voir Figure 10). Dans ces conditions, la stagiaire perd momentanément la main sur l'animation de son activité et se retrouve elle-même placée dans une position d'observatrice.

La suite de l'activité montre cependant que cette reconfiguration de la participation n'est que temporaire, et que les relances initiées par la référente vont elles-mêmes permettre à la stagiaire de se repositionner dans l'animation de son activité. L'extrait 6 intervient environ 15 minutes plus tard, au moment où les participants se sont repositionnés de manière différente dans l'environnement. La stagiaire est alors assise près du cylindre utilisé précédemment par la référente, et celle-ci fait rouler un ballon sur le toit du tunnel en effectuant des passes avec un enfant.

Extrait 6 «moi je connais pas la chanson » (54:58-56:00)

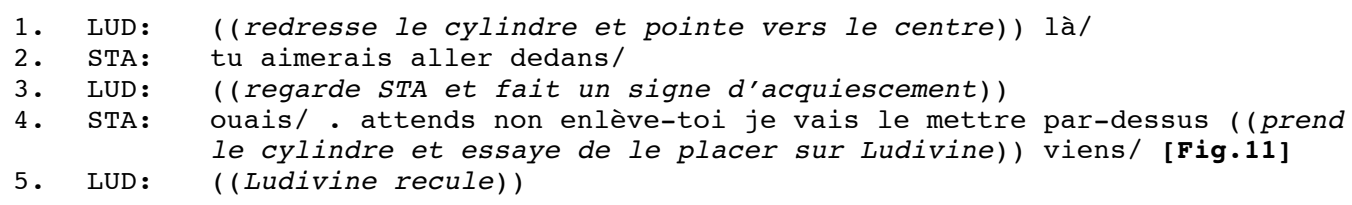


6. STA: ça fait peur hein/ ((repose le cylindre sur le sol)) viens je vais te mettre dedans comme çal ((prend Ludivine et la place dans le cylindre)) hop/

7. ALI: ( (Alix s'approche du cylindre et manifeste son envie d'aller à l'intérieur)) ah-li-li/ ah-li-li/

8. STA: toi aussi/

9. REF: li-li t'as vu comment elle s'appelle/ ((joue au ballon avec Thomas))

10. STA:

11. REF :

li-li ouais $\backslash$. tu t'appelles comment Alix

c'est dur à dire Alixl ((joue au ballon avec Thomas))

attention/ ((prend Alix et la place dans le cylindre)) bouge pas trop

les pieds/ . voilà : : :

((Maya accourt et veut aller dans le cylindre également))

encore/

encore en bateau/. moi je connais pas la chanson de REF mais

((chante en faisant tanguer le cylindre)) en bateau-Alix-et-Ludivineen-bateau-Alix-et-Ludivine-dans-l'eau/ quand-il-fait-de-gro : :ssesvagues-le-bateau-fait-plouf-dans-l'eau ((fait basculer le cylindre)) [Fig.12]

16. REF :

((REF observe la scène en souriant))

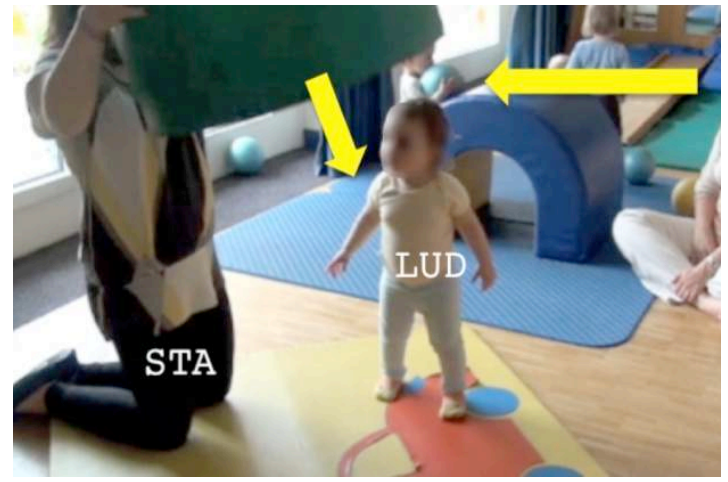

Figure $11:$ 00:55:04

STA essaye de placer le cylindre autour de LUD

Figure 11: STA tries to place the cylinder around $L U D$

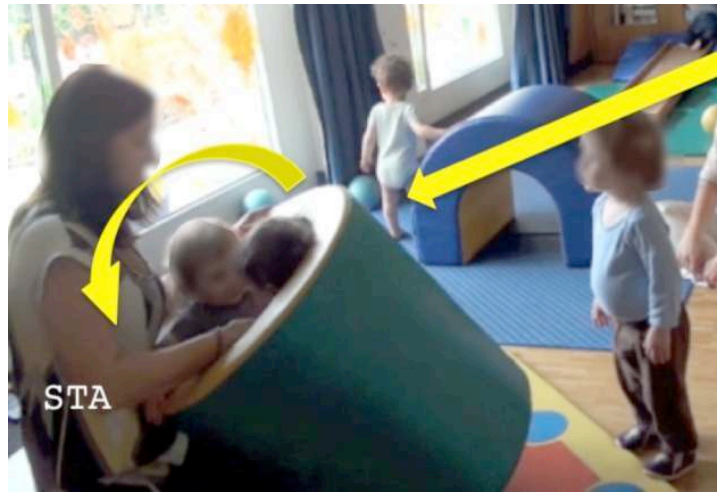

Figure $12: 00: 55: 47$

STA fait tanguer le cylindre en chantant la chanson du bateau ; REF observe en souriant

Figure 12: STA rocks the cylinder while singing the boat song; REF observes with a smile

Comme dans l'extrait 5, c'est ici à nouveau Ludivine qui manifeste de l'intérêt pour le cylindre et qui va contribuer à une reconfiguration de la participation de la stagiaire et de la référente dans la situation. En ligne 1, Ludivine s'approche du cylindre et tente de le remettre en position verticale. La stagiaire perçoit immédiatement son intention et en propose une reformulation («tu aimerais aller dedans/», ligne 2), ratifiée par Ludivine (1.3). La stagiaire va alors à la fois reproduire et transformer des stratégies d'animations précédemment accomplies par la référente et qui constituaient l'objet de sa monstration. Elle va commencer par placer Ludivine dans le centre du cylindre, mais en tentant la variante d'un portage du cylindre et non pas de l'enfant (voir Figure 11). Cette variante effraie la fillette, qui recule, obligeant la stagiaire à un retour à la technique originale («ça fait peur hein/ viens je vais te mettre dedans comme ça », ligne 6). Comme précédemment, le portage de Ludivine dans le cylindre visibilise un foyer d'activité qui attire l'attention d'autres enfants, comme Alix et Maya, qui accourent à nouveau vers le cylindre (ligne 7-13). L'animation peut alors se déployer dans un format analogue à celui accompli précédemment par la référente. La stagiaire place à son tour Alix au centre du cylindre, et, à la demande de Ludivine (« encore », ligne 14), commence à faire tanguer le cylindre en chantant la chanson du bateau (voir Figure 12). Elle fait d'ailleurs explicitement référence à la situation observée précédemment en signalant aux enfants qu'elle ne connaît pas bien la chanson (« encore en bateau/ moi je connais pas la chanson de REF mais », ligne 15). Cette remobilisation de ressources observées précédemment permet à la stagiaire de retrouver une place centrale dans l'animation de son activité, le tout sous le regard de la référente, qui se retrouve à son 
tour en position d'observatrice.

On voit donc dans ces deux derniers extraits comment la configuration de la monstration peut amorcer des prises de relais dans la co-animation de l'activité, et comment l'accompagnement tutoral peut emprunter, à certains moments, des formes d'ostensions qui agissent comme des ressources mimétiques pour les stagiaires. Ce format de participation confère un rôle momentanément dominant à la référente dans la conduite de l'activité éducative, mais suppose également de sa part des mises en retrait permettant à la stagiaire d'expérimenter les stratégies d'animation adressées et repérées. C'est donc sur ces formes d'alternances entre ostension et appropriation que résident à la fois les dynamiques propres et le potentiel formateur des configurations participatives de la monstration.

\section{5.- Vers des configurations de participation spécifiques au champ socio-éducatif ?}

Dans cet article, nous souhaitions, en continuité avec d'autres travaux, contribuer à extraire l'activité tutorale de l'anonymat et de «l'angle mort» dans lequel elle est encore souvent cantonnée. En particulier, à partir d'analyses d'interactions en situation de travail, nous avons cherché à décrire comment, dans le quotidien des institutions de la petite enfance, les référentes professionnelles aménagent pour les stagiaires débutantes des opportunités de participation à des activités éducatives, et réciproquement comment les stagiaires s'engagent dans ces opportunités pour expérimenter et développer les multiples compétences du métier d'éducatrice de l'enfance. Arrivés au terme de cette étape de nos investigations, quelques éléments peuvent être dégagés.

Un premier ensemble d'observations concerne à la fois la complexité et les spécificités de l'accompagnement tutoral en contexte éducatif. Nos analyses des données permettent de souligner que dans l'effervescence de leur action, les référentes professionnelles sont souvent engagées dans deux types d'activités : une activité de nature éducative auprès des jeunes enfants d'une part et une activité de nature formative auprès de la stagiaire d'autre part. Ces deux activités sont intimement liées et parfois difficiles à dissocier. Pour les discriminer, la didactique professionnelle a proposé de distinguer deux dimensions constitutives des activités humaines: les dimensions dites constructives et productives (Pastré, Mayen \& Vergnaud, 2006 ; Rabardel, 2005). La didactique professionnelle définit l'activité productive comme celle ayant pour effet des transformations du monde liées à la production de biens ou de services. L'activité constructive est celle que développe le sujet à l'occasion de son activité productive. C'est un aspect de l'expérience au sens d'une transformation de soi qui s'opère à l'occasion de la transformation du monde. Si, comme le postule la didactique professionnelle, il n'y a pas d'action sans pensée, les dimensions constructives de l'activité sont liées aux dimensions productives au sens où l'engagement dans l'action mobilise toujours une part subjective, celle de l'élaboration que l'on peut en faire dans le temps ou à contretemps de l'action. La distinction que nous avons proposée ici entre les enjeux éducatifs et de formation est d'une tout autre nature et renvoie aux formes complexes de cadrage de l'expérience qui caractérise les situations de formation par et dans le travail (Goffman, 1991). Il importe en effet de considérer que la conduite des activités éducatives aussi bien que celles en lien avec les enjeux de formation professionnelle présentent des dimensions à la fois productives et constructives. Celles-ci sont rendues manifestes par des transformations observables dans l'environnement matériel et amènent aussi les participants à mobiliser et transformer les répertoires de ressources nécessaires pour les conduire.

Comme permettent de le souligner nos analyses, les solutions pratiques apportées par les participants à l'interaction pour faire face à ce cadrage complexe de l'expérience sont multiples et prennent des formes variées et variables dans la dynamique de l'activité de travail. Il existe ainsi des moments où le cadre de l'activité formative se dissocie fortement de l'activité éducative et s'affiche comme un cadre «modalisateur» de celle-ci. C'est le cas 
par exemple de la configuration d'observation, dans laquelle l'activité éducative conduite par la stagiaire devient l'objet d'une activité d'évaluation à la fois distincte et conduite selon les prémisses organisationnelles spécifiques. À d'autres moments, l'activité formative se fond à l'intérieur du cadre éducatif «primaire » et se trouve en quelque sorte «modalisée »par celui-ci. C'est le cas par exemple des configurations de co-animation ou de monstration, qui rendent manifestes des dimensions formatives de l'activité, mais à partir de la conduite d'activités éducatives centrées sur les enfants. En d'autres termes, il apparaît que les modalités locales de participation des référentes présentent un caractère stratifié et donnent à voir les enjeux qu'elles attribuent à la situation selon la dynamique propre à l'activité. Selon la manière dont elles s'engagent dans les interactions, elles produisent des configurations de participation pour les stagiaires qui manifestent à la fois leur interprétation du cadre de l'activité tutorale et la manière dont elles endossent localement leur rôle de tutrice (Zimmerman, 1998). De ce point de vue, les trois configurations de participation identifiées peuvent être vues comme des réponses variées apportées par les participants pour faire face aux enjeux de cadrage complexe de l'expérience qui se manifestent dans des situations dans lesquelles se combinent à la fois des enjeux de travail et de formation.

À ce stade de nos recherches et comme nous l'avons illustré ci-dessus, trois configurations principales de participation à l'interaction se dégagent de nos observations, permettant de cerner des modalités spécifiques d'accomplissement de l'accompagnement tutoral: a) l'observation, b) la co-animation, c) la monstration. Ces formats d'accompagnement sont en partie distincts de ceux observés dans le contexte technique et artisanal par Kunégel (2005, 2011) ou dans nos propres travaux antérieurs portant sur la formation «duale » des apprentis (Filliettaz, 2010, 2011a, 2011b). À l'opposé d'une logique d'homogénéité et d'invariance, ces formats pointent surtout la grande variation des registres employés par les référentes pour exercer une fonction tutorale auprès des stagiaires dont elles assurent l'encadrement. Ces variations concernent en premier lieu la conduite des activités éducatives elles-mêmes, et les multiples configurations de participation qui peuvent s'y déployer de manière dynamique. Loin de constituer des formats d'accompagnement réifiés et clairement assimilables à un des binômes référente-stagiaire observés, elles apparaissent de façon souvent entremêlée pour chacun d'entre eux. Ces configurations sont donc indépendantes des binômes observés mais dépendantes des conditions émergentes de l'activité et des dynamiques de participation qui sont négociées entre les participants.

Ceci soulève un ensemble de questions qui méritent d'être posées. Existe-t-il une culture commune de l'accompagnement tutoral dans le champ de la petite enfance ? Ou, pour le dire dans les termes de la clinique de l'activité, existe-t-il, au-delà des déclinaisons et des variations de styles, un genre professionnel commun à l'activité des référentes professionnelles? Les quelques illustrations présentées ici ne permettent sans doute pas d'apporter des réponses définitives à de telles questions. En revanche, elles peuvent nous inciter à continuer de questionner le sens et les spécificités de ces pratiques, à collectiviser ces modes d'accompagnement et à rendre davantage visible, aux yeux des professionnels eux-mêmes, la complexité et la diversité des compétences interactionnelles mobilisées dans l'accomplissement de leur travail. Là réside probablement un des enjeux majeurs pour la professionnalisation des référentes professionnelles dans ce domaine socio-éducatif spécifique.

Ces questions prolongent et complètent des recherches conduites à propos des pratiques de transmission en situation de travail, à partir de démarches théoriques et méthodologiques distinctes de celles adoptées dans la présente recherche. Plusieurs recherches et interventions d'orientation ergonomique se sont en effet intéressées récemment à la problématique de l'accompagnement des novices en situation de travail, et ce dans des domaines professionnels variés, relevant tantôt de métiers techniques (le bâtiment, l'industrie alimentaire, la restauration) ou des services à la personne (les soins infirmiers). Ces travaux, tout comme ceux présentés ici, soulignent l'importance de l'accompagnement tutoral dans la mise en oeuvre des processus d'apprentissage au travail. Par exemple, Gaudart, Delgoulet et Chassaing (2008) montent comment, en dépit de son caractère aléatoire et peu formalisé, 
l'accompagnement tutoral joue un rôle déterminant dans les mécanismes de fidélisation des nouveaux employés dans les métiers du bâtiment. Cloutier, Ledoux \& Fournier (2012) aboutissent à des conclusions similaires, en relevant que des facteurs liés à l'organisation du travail, comme par exemple les emplois précaires ou encore l'intensification de la charge de travail, tendent à faire obstacle aux pratiques de transmission des savoirs entre experts et novices et contribuent de manière significative à l'appauvrissement des métiers.

Les travaux d'orientation ergonomique s'inscrivent également en continuité avec les résultats présentés ici en ce qu'ils contribuent à porter un regard renouvelé sur les « activités de transmission » elles-mêmes. Comme le relèvent aussi bien Gaudart \& Thébault (2012), que Ouellet (2012) et Kloetzer (2011), ces activités apparaissent comme fragiles, multifonctionnelles et sous contraintes. Elles doivent composer avec des logiques en tension, conciliant les exigences de production avec les rythmes propres à la formation et aux processus d'apprentissage. Enfin, comme le montrent bien les travaux de Gaudart, Delgoulet \& Chasseing (2008), les activités de transmission ne se ramènent pas à un processus unilatéral de communication d'un savoir expert en direction d'un destinataire novice. Elles impliquent au contraire une grande part de proactivité de la part des apprenants, qui doivent aller à la recherche de l'expertise, apprendre à observer et faire le tri entre les ressources disponibles. C'est là d'ailleurs que réside un écart souvent difficile à résorber entre les pratiques d'accompagnement telles qu'elles sont envisagées, prescrites et parfois idéalisées par les institutions de formation et les réalités de la formation telles qu'elles sont observables en situation de travail (Laberge, Vézina, Calvet, Lévesque, \& Vézina-Nadon, 2012).

Sur le plan méthodologique, les démarches d'inspiration ergonomique ont souvent visé une connaissance accrue et fine des pratiques réelles de transmission telles qu'elles sont attestées dans les organisations de travail. Cloutier, Ledoux \& Fournier (2012, p. 317) perçoivent d'ailleurs explicitement de telles études comme des pistes de recherches prometteuses permettant de mieux comprendre comment les experts en charge de la transmission des savoirs composent avec les attentes multiples et parfois contradictoires qui s'expriment à leur égard. En vue d'aboutir à une telle compréhension des pratiques réelles de transmission, une triangulation des données empiriques est souvent privilégiée, permettant de combiner l'analyse de documents à caractère prescriptif avec le recueil d'entretiens et la conduite d'observations directes en situation de travail. Les observations situées font souvent l'objet de prises de notes ou d'enregistrements audio (Ouellet, 2012). Parfois, elles donnent lieu aussi à des enregistrements vidéo, mais dont l'analyse tend à se centrer presque exclusivement sur les dimensions verbales de la communication entre experts et apprenants (Laberge et al., 2012). De ce point de vue, la perspective interactionnelle adoptée dans nos propres recherches permet d'entrevoir une exploitation approfondie du matériau audio-vidéo, qui ne se limite pas à une documentation des pratiques verbales, mais qui saisit les conduites situées dans leurs dimensions également corporelles, proxémiques et matérielles.

Enfin, les travaux de recherche et d'intervention réalisés dans le champ de l'ergonomie à propos des pratiques de transmission au travail soulignent de manière récurrente le besoin d'une formation accrue des tuteurs (Gaudart et al., 2008; Gaudart \& Thébault, 2012; Ouellet, 2012). Certains dispositifs de recherche se présentent d'ailleurs explicitement comme une contribution à de telles démarches. Par exemple, les travaux de Six-Touchard (1998) dans le champ de la formation professionnelle du personnel de cuisine visent clairement à outiller les tuteurs à l'analyse du contenu de leur travail, par la mise en place de dispositifs d'auto-analyse. Ces démarches d'auto-analyse produisent chez les tuteurs des prises de conscience qui se traduisent par des effets tangibles sur les contenus de l'interaction tutorale ainsi que sur les formes de guidage observées. Plus récemment, les travaux de Kloetzer (2011) ont proposé de recourir à des démarches d'instruction au sosie en clinique de l'activité pour accroitre le pouvoir d'agir des tuteurs. Ces démarches visent à soutenir un processus de développement non seulement individuel, mais aussi collectif, permettant de souligner les enjeux de «métier » qui se nouent autour de la pratique tutorale.

De telles démarches d'intervention et de formation invitent à des développements fructueux pour nos propres travaux. Dans le contexte suisse de la formation professionnelle, il existe à 
ce jour une offre de formation relativement fragmentée en direction des professionnelles de la petite enfance qui souhaitent développer ou faire reconnaître des compétences d'accompagnement tutoral. Des stages de formation continue de courte durée sont proposés, mais ceux-ci présentent la particularité de ne pas être certifiants et ne proposent pas un dispositif couvrant l'ensemble des compétences requises. À l'inverse, des formations certifiantes existent ${ }^{5}$, mais qui sont difficilement accessibles aux professionnelles de la petite enfance. Si quelques référentes professionnelles sont certes détentrices d'un certificat ciblé sur l'accompagnement des stagiaires dans le domaine social, la plupart ne le sont pas et souhaitent bénéficier d'une reconnaissance de leur statut. Les demandes de formation de la part des éducatrices endossant la fonction de tutrice sont nombreuses et les besoins largement reconnus par cette communauté qui soulève l'importance et l'intérêt à transmettre le métier. Face à ce constat, il apparaît qu'une étude visant à analyser l'activité des tuteurs au plus proche des situations de travail rencontrées peut contribuer à alimenter les dispositifs de professionnalisation existants. C'est là des besoins de formation exprimés par les acteurs eux-mêmes et relayés par les institutions qui les emploient. À côté d'apports théoriques, méthodologiques et organisationnels nécessaires sur la fonction tutorale, les professionnelles pointent l'intérêt d'être engagées dans des démarches d'analyse de leur activité en s'appuyant sur les traces réelles de leur activité par le biais d'enregistrements audio-vidéo. Sur ce point, des expériences récentes menées par des membres de notre équipe avec les collectifs de professionnelles engagés dans la recherche ont été réalisées à titre exploratoire. En nous appuyant sur un dispositif d'auto et d'inter-confrontation vidéo (Leclercq, 2005), nous avons ré-exploité à des fins de formation des séquences mettant en évidence différents formats d'accompagnement tutoral. Les premiers résultats de cette transposition sont prometteurs et laissent entrevoir des liens fructueux entre le repérage analytique des configurations de participation à l'interaction tutorale et la possibilité pour les professionnelles d'une connaissance et d'une reconnaissance accrues de leur activité.

\section{BIBLIOGRAPHIE}

Barbier, J.-M. (1996). Tutorat et fonction tutorale. Recherche et formation, 22, 7-19.

Billett, S. (2001). Learning in the workplace: strategies for effective practice. Crows Nest: Allen and Unwin.

Billett, S. (2009). Modalités de participation au travail : la dualité constitutive de l'apprentissage par le travail. In M. Durand, \& L. Filliettaz (Eds.), Travail et formation des adultes (pp. 37-63). Paris: Presses universitaires de France.

Bruner, J. (1983). Le développement de l'enfant. Savoir faire, savoir dire. Paris: Presses Universitaires de France.

Cloutier, E., Ledoux, E., \& Fournier, P.-S. (2012). Knowledge transmission in light of recent transformations in the workplace. Industrial Relations, 67(2), 304-324.

Clot, Y. (2000). Analyse psychologique du travail et singularité de l'action. In J.-M. Barbier (Ed.), L'analyse de la singularité de l'action (pp. 53-69). Paris: PUF.

Duc, B. (2012). La transition de l'école au monde du travail : une analyse interactionnelle et longitudinale des phénomènes de participation et de construction identitaire en formation professionnelle initiale. Thèse de doctorat en Sciences de l'éducation, Genève: Faculté de Psychologie et des Sciences de l'Éducation, Université de Genève.

Duranti, A., \& Goodwin, C. (Ed.) (1992). Rethinking context: language as an interactive phenomenon. Cambridge: Cambridge University Press.

Filliettaz, L. (2009). Les discours de consignes en formation professionnelle initiale : une approche linguistique et interactionnelle. Éducation \& Didactique, 3(1), 91-111.

Filliettaz, L. (2010). Guidance as an interactional accomplishment: Practice-based learning within the Swiss VET system. In S. Billett (Ed.), Learning through practice: Models, traditions, orientations

5 Dans le contexte de la Suisse, l'Institut Fédéral des hautes études en Formation Professionnelle (IFFP) délivre des certificats d'études avancées de formateur à la pratique professionnelle dans les études supérieures. Notons par ailleurs qu'au niveau HES (haute école spécialisée), des certificats d'études avancées de praticien-formateur sont également délivrés dans les domaines de la santé et du travail social. 
and approaches (pp. 156-179). Dordrecht: Springer.

Filliettaz, L. (2011a). Asking questions... getting answers. A sociopragmatic approach to vocational training interactions. Pragmatics \& Society, 2(2), 234-259.

Filliettaz, L. (2011b). Collective guidance at work: a resource for apprentices? Journal of Vocational Education and Training, 63(3), 485-504.

Filliettaz, L. (à paraître a). L'interaction langagière : un objet et une méthode d'analyse en formation d'adultes. In J. Friedrich, \& J. Pita (Eds.), Un dialogue entre concepts et réalité. Dijon: Éditions Raisons et Passions.

Filliettaz, L. (à paraître b). «C'est chacun son tour » : la mise en visibilité de la structuration de l'interaction en contexte d'éducation et de formation. In J. Jacquin, \& X. Gradoux (Eds.), Le niveau méso-interactionnel, lieu d'articulation entre langage et activité. Cahiers de l'ILSL: Université de Lausanne.

Filliettaz, L. (à paraître c). Learning through interactional participatory configurations: Contributions from video analysis. In Ch. Harteis, A. Rausch, \& J. Seifried (Eds.), Discourses of Professional Learning: On the Boundary between Learning and Working. Dordrecht: Springer.

Filliettaz, L., \& Rémery, V. (à paraître). Transmettre le travail par les mises en forme langagières de l'activité. In R. Wittorski (Ed.), Transmettre le travail. Paris: L'Harmattan.

Filliettaz, L., de Saint-Georges, I., \& Duc, B. (2008). «Vos mains sont intelligentes! » Interactions en formation professionnelle initiale. Université de Genève: Cahiers de la Section des Sciences de l'Éducation, 117.

Filliettaz, L., de Saint-Georges, I., \& Duc, B. (2009). Interactions et dynamiques de participation en formation professionnelle initiale. In M. Durand, \& L. Filliettaz (Eds.), Travail et formation des adultes (pp. 95-124). Paris: Presses Universitaires de France.

Fuller, A., \& Unwin, L. (2003). Learning as apprentices in the contemporary UK workplace: Creating and managing expansive and restrictive participation. Journal of Education and Work, 16(4), 407426.

Gaudart, C., Delgoulet, C., \& Chassaing, K. (2008). La fidélisation de nouveaux dans une entreprise du BTP : Approche ergonomique des enjeux et des déterminants. Activités, 5(2), 2-24. http://www.activites.org/v5n2/v5n2.pdf.

Gaudart, C., \& Thébault, J. (2012). La place du care dans la transmission des savoirs professionnels entre anciens et nouveaux à l'hôpital. Relations industrielles, 67(2), 242-262.

Goffman, E. (1973). La mise en scène de la vie quotidienne. Tome 1 : La présentation de soi. Paris: Éditions de Minuit.

Goffman, E. (1987). La position. In Façons de parler (pp. 133-166). Paris: Éditions de Minuit.

Goffman, E. (1991). Les cadres de l'expérience. Paris: Minuit.

Heath, Ch., Knoblauch, H., \& Luff, P. (2000). Technology and social interaction: the emergence of "workplace studies". British Journal of Sociology, 51(2), 299-320.

Kloetzer L. (2011). L'engagement conjoint dans la pratique comme clef du développement de l'activité des tuteurs. ePTO, 17, 445-464.

Kunégel, P. (2005). L'apprentissage en entreprise : l'activité de médiation des tuteurs. Éducation permanente, 165, 127-138.

Kunégel, P. (2011). Les maîtres d'apprentissage. Analyse des pratiques tutorales en situation de travail. Paris: L'Harmattan.

Lamamra, N., \& Masdonati, J. (2009). Arrêter une formation professionnelle : mots et maux d'apprenti-e-s. Lausanne: Antipodes.

Laberge, M., Vézina, N., Calvet, B., Lévesque, S., \& Vézina-Nadon, L. (2012). Supervision of Apprentices in Semiskilled Trades: Program Stipulations and Workplace Realities. Industrial Relations, 67(2), 199-221.

Lave, J. \& Wenger, E. (1991). Situated learning: legitimate peripheral participation. Cambridge: Cambridge University Press.

Leclercq, G. (2005). Les dispositifs de formation professionnalisés comme situation d'auto et d'interconfrontation. L'impact d'un environnement numérique de travail. Les institutions éducatives face au numérique. Actes du colloque du SIF, MSH Paris Nord. 
Markaki, V., \& Rémery, V. (2013). Les processus de hiérarchisation dans l'activité tutorale au travail. Contribution au Congrès de l'Association Française de Sociologie, Nantes, 2-5 septembre 2913.

Mayen, P. (2002). Le rôle des autres dans le développement de l'expérience. Éducation permanente, 151, 87-107.

Mondada L. (2006), Interactions en situations professionnelles et institutionnelles : de l'analyse détaillée aux retombées pratiques. Revue française de linguistique appliquée, XI(2), 5-16.

Olry, P., \& Cuvillier, B. (2007). Apprendre en situation. Le cas des dispositifs apprentis/maîtres d'apprentissage. Éducation Permanente, 17, 3.

Olry-Louis, I., \& Olry, P. (2011). Transmettre la relation de service par l'interaction tutorale : le cas des facteurs. Psychologie du Travail et des Organisation, 17(3), 269-289.

Ouellet, S. (2012). Workplace Training: How Context Impacts on Instructors' Activities. Industrial Relations, 67(2), 222-241.

Pastré, P., Mayen, P., \& Vergnaud, G. (2006). La didactique professionnelle. Revue Française de Pédagogie, 154, 145-198.

Rabardel, P. (2005). Activité constructive, productive, apprentissage. In P. Pastré, \& P. Rabardel (Eds.), Modèles du sujet pour la conception. Toulouse: Octarès Éditions

Savoyant, A. (1995). Guidage de l'activité et développement des compétences dans une entreprise d'insertion. Éducation permanente, 123, 91-99.

Savoyant, A. (1996). Une approche cognitive de l'alternance. Bref, Cereq, nº 118.

Six-Touchard, B. (1998). Développement de la compétence tutorale par l'auto-analyse du travail. Éducation Permanente, 138(2), 87-98.

Stalder, B.E., \& Nägele, C. (2011). Vocational education and training in Switzerland: Organisation, development and challenges for the future. In M. M. Bergman, S. Hupka-Brunner, A. Keller, T. Meyer, \& B.E. Stalder (Eds.), Youth transitions in Switzerland: Results from the TREE panel study (pp. 18-39). Zürich: Seismo.

Theureau, J. (2000). Anthropologie cognitive et analyse des compétences. In J.-M. Barbier (Ed.), L'analyse de la singularité de l'action (pp. 171-211). Paris: PUF.

Veillard, L., \& Kouamé Kouassi, D. (2012). Concevoir un outil d'aide au transfert de connaissances en formation par alternance. Travail \& Apprentissage, 9, 41-61.

Vermersch, P. (1994). L'entretien d'explicitation. Paris: ESF.

Wenger, E. (1998). Community of practice: Learning, meaning, and identity. Cambridge: Cambridge University Press.

Zimmerman, D.H. (1998). Identity, context and interaction. In C. Antaki, \& S. Widdicombe (Eds.), Identities in talk (pp. 87-106). London: Sage.

Zogmal, M., Losa, S., \& Filliettaz, L. (2013). Le travail de structuration des activités menées auprès des enfants. Revue Petite Enfance, 111, 92-111.

\section{ANNEXE : CONVENTIONS DE TRANSCRIPTION}

I

1

BOUteille

:

(incertain)

XXX

......

souligné

$\mathrm{REF}>\mathrm{STA}$

((commentaire))

[Fig.1]

\section{intonation montante}

intonation descendante

accentuation

allongements syllabiques

troncations

segments dont la transcription est incertaine

segments inaudibles

pauses de durée variable

chevauchements dans les prises de parole

relation d'allocution directe (désignation de l'interlocuteur principal) commentaire du transcripteur, relatif à des conduites gestuelles ou des actions non verbales

index renvoyant à la position de l'image dans la transcription 


\section{RÉSUMÉ}

Cette contribution se propose de mieux comprendre comment s'y prennent des éducatrices de l'enfance endossant une fonction tutorale en situation de travail pour encadrer et accompagner des étudiantes stagiaires au début de leur formation professionnelle. À partir d'une analyse interactionnelle et multimodale de l'activité qui se déploie en situation de travail avec des groupes d'enfants, nous proposons d'étudier comment se construisent et se négocient entre les tutrices et les stagiaires des configurations de participation dans ces situations de formation sur la place de travail. Comment, dans le quotidien du travail dans les institutions de la petite enfance, les tutrices aménagent-elles pour les stagiaires des opportunités de participation à des activités éducatives? Et réciproquement, comment les stagiaires investissent-elles ces opportunités de participation pour expérimenter, sous différentes facettes, les compétences professionnelles visées dans leur parcours de formation? L'analyse d'enregistrements audio-vidéo réalisés en situation naturelle de travail permet de mettre en évidence trois configurations de participation principales : a) l'observation, b) la co-animation, c) la monstration. Ces configurations permettent de cerner non seulement des formats interactionnels différents, mais encore les transformations dynamiques qui caractérisent la relation tutorale dans les conditions réelles de son accomplissement.

\section{MOTS CLÉS}

tutorat, apprentissage au travail, interaction, participation, étayage

\section{RÉFÉRENCEMENT}

Filliettaz, L., Rémery, V., \& Trébert, D. (2014). Relation tutorale et configurations de participation à l'interaction: Analyse de l'accompagnement des stagiaires dans le champ de la petite enfance. Activités, 11(1), 22-46, http://www.activites.org/v11n1/v11n1.pdf

Article soumis le 3 mai 2013, accepté pour publication le 2 décembre 2013. 